\title{
Higher-dimensional invariants in 6D super Yang-Mills theory
}

\author{
Serafim Buyucli ${ }^{1}$ and Evgeny Ivanov ${ }^{a, b}$ \\ ${ }^{a}$ Bogoliubov Laboratory of Theoretical Physics, JINR, \\ Dubna 141980, Moscow region, Russia \\ ${ }^{b}$ Moscow Institute of Physics and Technology, \\ Dolgoprudny 141700, Moscow region, Russia \\ E-mail: eivanov@theor.jinr.ru
}

Abstract: We exploit the $6 D, \mathscr{N}=(1,0)$ and $\mathscr{N}=(1,1)$ harmonic superspace approaches to construct the full set of the maximally supersymmetric on-shell invariants of the canonical dimension $\mathbf{d}=\mathbf{1 2}$ in $6 D, \mathscr{N}=(1,1)$ supersymmetric Yang-Mills (SYM) theory. Both single- and double-trace invariants are derived. Only four single-trace and two double-trace invariants prove to be independent. The invariants constructed can provide the possible counterterms of $\mathscr{N}=(1,1)$ SYM theory at four-loop order, where the first double-trace divergences are expected to appear. We explicitly exhibit the gauge sector of all invariants in terms of $\mathscr{N}=(1,0)$ gauge superfields and find the absence of $\mathscr{N}=(1,1)$ supercompletion of the $F^{6}$ term in the abelian limit.

KEywords: Extended Supersymmetry, Superspaces, Supersymmetric Gauge Theory

ARXiv EPrint: 2105.05899

\footnotetext{
${ }^{1}$ Deceased.
} 


\section{Contents}

1 Introduction 1

$2 \quad 6 D$ spinor algebra and HSS 3

$3 \mathscr{N}=(1,0)$ supersymmetric Yang-Mills theory $\quad 7$

$\begin{array}{lll}3.1 \text { Kinematics } & 7\end{array}$

3.2 Dynamics 8

$4 \mathscr{N}=(1,1)$ SYM in bi-HSS formulation $\quad 10$

5 On-shell $\mathscr{N}=(1,1)$ SYM invariants $\quad 13$

$\begin{array}{lll}5.1 \text { Generalities } & 13\end{array}$

$\begin{array}{lll}5.2 & \text { Examples } & 15\end{array}$

$6 \quad$ Invariants of dimension $d=12 \quad 17$

$\begin{array}{lll}6.1 & \text { Invariants without derivatives } & 18\end{array}$

$\begin{array}{lll}6.2 & \text { Invariants with derivatives } & 19\end{array}$

7 Passing to $\mathscr{N}=(1,0)$ superfields $\quad 21$

8 Summary and outlook $\quad 26$

$\begin{array}{ll}\text { A Some integrals } & 26\end{array}$

\section{Introduction}

Maximally supersymmetric theories in diverse dimensions are of particular interest in view of their close relation to superstrings and D-branes. This concerns, e.g., supersymmetric Yang-Mills (SYM) theories with sixteen supercharges existing in dimensions $D \leq 10$. In particular, maximally supersymmetric $6 D, \mathscr{N}=(1,1)$ SYM theory arises as a low-energy effective field theory on coincident D5-branes and is reduced to $4 D, \mathscr{N}=4 \mathrm{SYM}$ theory upon the appropriate compactification $6 D \rightarrow 4 D$. The study of quantum $6 D, \mathscr{N}=(1,1)$ SYM theory attracts a heightened attention despite the fact that this theory is nonrenormalizable by power-counting because of dimensionful coupling constant. The relevant full quantum effective action is expected to provide an ultra violet (UV) completion of this theory and to be identical, after summing up all loop contributions, to the full Born-Infeld-type world-volume action of D5-branes [1].

The quantum structure of the maximally extended gauge and supergravity theories is uncovered by studying their amplitudes, for which various powerful methods have been developed, see, e.g., refs. [2-16] (a more complete list of references can be found in the paper [15]). Using these methods, some surprising features of $6 D, \mathscr{N}=(1,1) \mathrm{SYM}$ theory in the quantum domain were observed. For instance, it is free of logarithmic divergences 
up to two loops, at three loops such divergences are seen only in the sector of planar (single-trace) diagrams, while the non-planar (double-trace) diagrams are finite.

No off-shell superfield formalism for $6 D, \mathscr{N}=(1,1)$ SYM theory is known, while such a formulation was developed for $6 D, \mathscr{N}=(1,0)$ SYM theory in $[17,18]$ in the framework of the conventional $6 D, \mathscr{N}=(1,0)$ superspace. The harmonic superspace (HSS) approach suggested earlier for $4 D, \mathscr{N}=2$ theories $[19,20]$ was generalized to six dimensions in refs. [21, 22]. The higher-dimensional counterterms of $\mathscr{N}=(1,0)$ and $\mathscr{N}=(1,1) \mathrm{SYM}$ theories were studied in conventional $6 D, \mathscr{N}=(1,0)$ superspace $[23,24]$ and in $6 D$ HSS, as well as in its on-shell extension, bi-HSS with two sets of $\mathrm{SU}(2)$ harmonic variables $[15$, $25,26]^{1}$

The quantum (Wilsonian) effective action of $6 D, \mathscr{N}=(1,1)$ SYM theory probably involves an infinite set of counterterms with the higher-order derivatives. Generically, they are not supersymmetric off shell and need not to be supersymmetric even on the shell of original equations of motion. Since every new term added to the original action can be looked upon as a deformation of the latter, the form of the "on-shell" supersymmetry transformations can vary from one loop order to another. It is the effective action with the whole set of counterterms that is expected to possess the properly realized deformed $\mathscr{N}=(1,1)$ supersymmetry. As was argued in [28], up to the fifth order in loops it is legitimate to deal with the transformations in which the superfields involved are on the shell of the equations of motion corresponding to the original "microscopic" action. The deformation effects would manifest themselves only in the next orders. The corresponding canonical (scaling) dimensions of the component Lagrangians are even and can vary from $\mathbf{d}=\mathbf{6}$ (one loop) to $\mathbf{d}=\mathbf{1 4}$ (five loops).

Possible higher-dimensional counterterms were explicitly constructed in [26] using the off-shell $6 D, \mathscr{N}=(1,0)$ and the on-shell $\mathscr{N}=(1,1)$ harmonic superspace approaches. For the canonical dimension $\mathbf{d}=\mathbf{6}$, all $6 D, \mathscr{N}=(1,1)$ invariants were shown to vanish on shell, which amounts to the one-loop finiteness of $\mathscr{N}=(1,1)$ SYM theory. The non-trivial onshell $\mathscr{N}=(1,1)$ supersymmetric and gauge invariant $\mathbf{d}=\mathbf{8}$ operators exist, though they do not possess the full off-shell $\mathscr{N}=(1,0)$ supersymmetry required by the perturbation theory in $6 D, \mathscr{N}=(1,0) \mathrm{HSS}^{2}$ Hence, the theory is UV finite up to two loops and UV divergences should start from three loops, in accord with the general reasoning of [14]. The invariants, corresponding to the three-loop order of the perturbation expansion and having dimension $\mathbf{d}=\mathbf{1 0}$, are of the two types, planar (single-trace) and non-planar (doubletrace). An interesting property of the single-trace invariant is that it can be equivalently written as an integral over the full bi-HSS, as well as over its $3 / 4$ analytic subspace. The double-trace invariant can be defined only in the analytic subspace. This may be one of the reasons why the double-trace invariant is UV protected and does not make contribution to the three-loop amplitude that was revealed by explicit calculations in [8-13].

In this paper we continue the analysis of ref. [26] and construct the maximally supersymmetric on-shell invariants of $6 D, \mathscr{N}=(1,1)$ SYM theory with the canonical dimension

\footnotetext{
${ }^{1} \mathrm{~A}$ similar bi-harmonic on-shell formulation of $4 D, \mathscr{N}=4 \mathrm{SYM}$ was recently worked out in [27].

${ }^{2}$ The manifestly off-shell $6 D, \mathscr{N}=(1,0)$ supersymmetric and gauge covariant quantum superfield technique in $6 D$ HSS was worked out and applied in [29-31].
} 
$\mathbf{d}=\mathbf{1 2}$ which should be responsible for the four-loop divergences. We find that there are four single-trace invariants and only two double-trace invariants of this dimension. Unlike the $\mathbf{d}=\mathbf{1 0}$ invariants, it is impossible to equivalently rewrite any of the $\mathbf{d}=\mathbf{1 2}$ ones as integrals over the analytic subspaces of $6 D, \mathscr{N}=(1,1)$ HSS, with the proper manifestly analytic Lagrangian densities.

In section 2 we start with recalling basic features of the spinor $6 D$ formalism and of $\mathscr{N}=(1,0)$ HSS with one set of SU(2) harmonic variables and $\mathscr{N}=(1,1)$ HSS with two sets of such variables. The brief exposition of the HSS description of $\mathscr{N}=(1,0)$ SYM theory and $6 D$ hypermultiplets is a subject of section 3 . The on-shell constraints of $\mathscr{N}=(1,1)$ SYM theory in the bi-HSS and their solution in terms of $\mathscr{N}=(1,0)$ harmonic superfields found in [26] are reviewed in section 4 . In section 5 we recall the $\mathscr{N}=(1,1)$ HSS form of the on-shell invariants constructed in [26] up to the dimension $\mathbf{d}=\mathbf{1 0}$. We present a new double-trace $\mathbf{d}=\mathbf{1 0}$ invariant in which both $\mathscr{N}=(1,0)$ and $\mathscr{N}=(0,1)$ supersymmetries are realized on shell. The structure of new $\mathbf{d}=\mathbf{1 2} \mathscr{N}=(1,1)$ invariants is discussed in detail in section 6 . In section 7 we consider the reduction of these invariants to $\mathscr{N}=(1,0)$ SYM invariants. We also study the abelian limit of the new invariants and show that exists no abelian $\mathscr{N}=(1,1)$ completion of the bosonic $F^{6}$ invariant. Some technical details are brought in appendix.

This paper is partially based on the bachelor thesis of Serafim Buyucli, the fourthgrade student of MIPT, defended by him in JINR under the supervision of E. Ivanov in June of 2018. Serafim had died tragically in August of 2018.

\section{$2 \quad 6 D$ spinor algebra and HSS}

We start by recalling generalities of $6 D$ spinor algebra, as well as of $\mathscr{N}=(1,0)$ and $\mathscr{N}=(1,1)$ ordinary and harmonic superspaces (HSS). More details can be found in $[17,19-$ $22,26,32]$. We will basically follow the notation and conventions of [26].

The group $\operatorname{Spin}(5,1)$ has two inequivalent irreducible spinor representations of complex dimension 4 , that is $(1,0)$ and $(0,1)$ spinors. In contrast to $4 D$ case, the complex conjugation does not interchange them, but produces an equivalent representation. In what follows, we will deal with the symplectic Majorana-Weyl spinors $\lambda_{i}^{a}$ and $\psi_{a A}$ carrying extra $\mathrm{SU}(2) \simeq \mathrm{Sp}(1)$ indices $i=1,2 ; A=1,2$ and satisfying the pseudoreality conditions, e.g.,

$$
\overline{\lambda_{i}^{a}}=-C_{b}^{a}\left(\lambda_{i}^{b}\right)^{*}=\varepsilon^{i k} \lambda_{k}^{a},
$$

$C$ being the charge conjugation matrix $\left(C=-C^{T}, C^{2}=-1\right)$. The generators of the spinor representations are $S^{M N}=-\frac{1}{2} \sigma^{M N}$, with

$$
\left(\sigma^{M N}\right)_{b}^{a}=\frac{1}{2}\left(\tilde{\gamma}^{M} \gamma^{N}-\tilde{\gamma}^{N} \gamma^{M}\right)_{b}^{a}=\frac{1}{2}\left(\gamma^{N} \tilde{\gamma}^{M}-\gamma^{M} \tilde{\gamma}^{N}\right)_{b}^{a}, \quad M, N=0, \ldots 5,
$$

where gamma matrices $\left(\gamma^{M}\right)_{a c}$ and $\left(\tilde{\gamma}^{N}\right)^{c b}$ are antisymmetric $4 \times 4$ matrices satisfying the $6 D$ Clifford algebra relation

$$
\left(\gamma^{M}\right)_{a c}\left(\tilde{\gamma}^{N}\right)^{c b}+\left(\gamma^{N}\right)_{a c}\left(\tilde{\gamma}^{M}\right)^{c b}=-2 \delta_{a}^{b} \eta^{M N}, \quad \eta^{M N}=\operatorname{diag}(1,-1,-1,-1,-1,-1),
$$


and

$$
\left(\tilde{\gamma}_{M}\right)^{a b}=\frac{1}{2} \varepsilon^{a b c d}\left(\gamma_{M}\right)_{c d}, \quad \varepsilon_{1234}=\varepsilon^{1234}=1 .
$$

Some other useful properties of gamma matrices (employed in section 7) are

$$
\begin{aligned}
\left(\gamma_{M}\right)_{a b}\left(\gamma_{M}\right)_{c d} & =2 \varepsilon_{a b c d}, \quad\left(\tilde{\gamma}_{M}\right)^{a b}\left(\tilde{\gamma}_{M}\right)^{c d}=2 \varepsilon^{a b c d}, \\
\left(\sigma^{M N}\right)_{b}^{a}\left(\sigma_{M N}\right)_{d}^{c} & =2\left(\delta_{b}^{a} \delta_{d}^{c}-4 \delta_{d}^{a} \delta_{b}^{c}\right), \\
\operatorname{Tr}\left(\sigma^{M N} \sigma^{S T}\right) & =4\left(\eta^{N S} \eta^{M T}-\eta^{M S} \eta^{N T}\right) .
\end{aligned}
$$

In the spinor notation, $6 D$ vectors are uniquely represented as

$$
V_{M} \leftrightarrow V_{a b}:=\frac{1}{2}\left(\gamma^{M}\right)_{a b} V_{M}, \quad V_{M}=\frac{1}{2} \tilde{\gamma}_{M}^{a b} V_{a b}
$$

Respectively, $6 D$ Minkowski space can be parameterized by the coordinates $x^{a b}$ related to the standard $6 D$ coordinates $X^{M}, M=0, \ldots, 5$ as

$$
x^{a b}=\left(\tilde{\gamma}^{M}\right)^{a b} x_{M}, \quad \partial_{a b}=\frac{1}{2}\left(\gamma^{M}\right)_{a b} \partial_{M} .
$$

In what follows, we shall make use of the superspace approach in which $6 D$ Minkowski space is extended by anticommuting Grassmann odd spinor coordinates $\theta_{i}^{a}$ and $\theta_{a A}$, with $i=1,2 ; A=1,2$ being doublet indices of some external automorphism ( $R$-symmetry) $\mathrm{SU}(2)$ groups.

The standard superspace approach is not too useful for gauge theories with extended supersymmetry. First, it does not allow to reveal the basic unconstrained objects of the theory and to construct the relevant off-shell superfield actions. Secondly, the standard superspace constraints on the basic gauge superfields frequently put the theory on mass shell and give no simple hints as to which portion of the total supersymmetry still admits an off-shell realization. Much more suggestive is the harmonic superspace (HSS) approach firstly discovered for $4 D, \mathscr{N}=4$ supersymmetry in [19, 20]. Off-shell superfield formulations of both $6 D, \mathscr{N}=(1,0)$ SYM theory and hypermultiplets become possible within $6 D, \mathscr{N}=(1,0)$ HSS [21, 22], a direct generalization of $4 D, \mathscr{N}=2$ HSS. On the other hand, in an analogous formulation of the maximally extended $\mathscr{N}=(1,1)$ SYM theory in $6 D$ HSS with the double set of harmonic and Grassmann variables $[15,26]$ the total $\mathscr{N}=(1,1)$ Poincaré supersymmetry admits a closure on the corresponding harmonic superfields only on shell. Hence, the maximally supersymmetric $\mathscr{N}=(1,1)$ invariants can also be defined on shell (though they can still respect off-shell $\mathscr{N}=(1,0)$ supersymmetry).

Now we sketch the basic notions of $\mathscr{N}=(1,0)$ HSS. The $\mathscr{N}=(1,0)$ super Poincaré group in its bosonic sector involves the $\mathrm{SU}(2)$ automorphisms ( $R$-symmetry) group, besides $6 D$ Poincare group. When defining the standard $\mathscr{N}=(1,0)$ superspace, this $\mathrm{SU}(2)$ is factored out, so this superspace is parameterized by the coordinate set

$$
z:=\left(x^{a b}, \theta_{i}^{c}\right) .
$$

$\mathscr{N}=(1,0)$ HSS is obtained by factoring out only the $\mathrm{U}(1)$ part of the automorphism $\mathrm{SU}(2)$. Thus each superspace point is equipped with the additional harmonic variables 
$u^{ \pm i}$ which are the coordinates of the sphere $S^{2} \simeq \mathrm{SU}(2) / \mathrm{U}(1), u^{+i} u_{i}^{-}=1$. This extended coordinate set yields the central basis of $\mathscr{N}=(1,0) \mathrm{HSS}$

$$
Z:=(z, u)=\left(x^{a b}, \theta_{i}^{c}, u^{ \pm i}\right) .
$$

It is convenient to define an analytic basis in this HSS, by passing to the coordinates

$$
Z^{(a n)}:=\left(x^{(a n)(a b)}, \theta^{ \pm c}, u^{ \pm i}\right)
$$

where

$$
x^{(a n) a b}=x^{a b}+\frac{i}{2}\left(\theta^{+a} \theta^{-b}-\theta^{+b} \theta^{-a}\right), \quad \theta^{ \pm a}=u_{k}^{ \pm} \theta^{a k} .
$$

Now $\mathscr{N}=(1,0)$ supersymmetry transformations prove to be closed also on the reduced set of coordinates

$$
\zeta:=\left(x^{(a n) a b}, \theta^{+c}, u^{ \pm i}\right),
$$

that parametrize a Grassmann-analytic subspace. ${ }^{3}$ Since G-analytic superfields depend only on the half of odd variables, their component structure is simpler than that of general harmonic superfields. In order to define the G-analyticity condition, we introduce the spinor differential operators

$$
D_{a}^{+}=\partial_{-a}, \quad D_{a}^{-}=-\partial_{+a}-2 i \theta^{-b} \partial_{a b}, \quad\left\{D_{a}^{+}, D_{b}^{-}\right\}=2 i \partial_{a b},
$$

which are related to the spinor derivatives in the central basis (2.9) by the standard relations, $D_{a}^{ \pm}=D_{a}^{i} u_{i}^{ \pm}$. Then an arbitrary G-analytic superfield $\Phi(\zeta)$ can be defined as the one satisfying the Grassmann analyticity condition

$$
D_{a}^{+} \Phi=0 .
$$

Like in $4 D$ case [20], the existence of the analytic harmonic superspace with the half set of Grassmann coordinates and the opportunity to define "short" harmonic analytic superfields are the pivotal merits of the $6 D, \mathscr{N}=(1,0)$ HSS approach.

The presence of additional bosonic coordinates brings about new algebraic relations, those involving the harmonic derivatives,

$$
\left[D^{++}, D^{--}\right]=D^{0},
$$

where (in the analytic basis)

$$
\begin{aligned}
D^{ \pm \pm} & =\partial^{ \pm \pm}+i \theta^{+a} \theta^{+b} \partial_{a b}+\theta^{+a} \partial_{-a}, \quad \partial^{ \pm \pm}=u^{ \pm i} \frac{\partial}{\partial u^{\mp i}} \\
D^{0} & =u^{+i} \frac{\partial}{\partial u^{+i}}-u^{-i} \frac{\partial}{\partial u^{-i}}+\theta^{+a} \partial_{+a}-\theta^{-a} \partial_{-a} .
\end{aligned}
$$

The commutation relations between harmonic and spinor derivatives can be easily established

$$
\left[D^{++}, D_{a}^{+}\right]=\left[D^{--}, D_{a}^{-}\right]=0, \quad\left[D^{++}, D_{a}^{-}\right]=D_{a}^{+}, \quad\left[D^{--}, D_{a}^{+}\right]=D_{a}^{-} .
$$

\footnotetext{
${ }^{3}$ Both coordinate sets (2.10) and (2.12) are real under the generalized conjugation defined in [20], so the latter is indeed a subspace of the former.
} 
As was said earlier, an on-shell superfield description of $6 D, \mathscr{N}=(1,1)$ SYM theory is achieved in the framework of bi-harmonic $\mathscr{N}=(1,1)$ superspace. It is an extension of the superspace $(2.9)$ by new right-handed $\operatorname{Spin}(5,1)$ spinors $\theta_{a A}$, where on the doublet indices $A=1,2$ an additional $R$-symmetry $\mathrm{SU}(2)$ group acts, and by the corresponding extra set of harmonics $u_{A}^{\hat{ \pm}}, u^{\hat{+} A} \hat{\bar{A}}=1$,

$$
\begin{aligned}
& Z \Rightarrow \hat{Z}:=\left(x^{(a n) a b}, \theta^{ \pm a}, \theta_{a}^{\hat{ \pm}}, u_{i}^{ \pm}, u_{A}^{\hat{ \pm}}\right), \\
& \theta_{a}^{\hat{ \pm}}=\theta_{a}^{A} u_{A}^{\hat{ \pm}}, \quad x^{(a n) a b}=x^{a b}+\frac{i}{2}\left(\theta^{+a} \theta^{-b}-\theta^{+b} \theta^{-a}\right)+\frac{i}{2} \varepsilon^{a b c d} \theta_{c}^{\hat{+}} \theta_{d}^{\hat{-}} .
\end{aligned}
$$

In this basis, both spinor derivatives $D_{a}^{+}$and $D^{\hat{+} a}$ are short,

$$
D_{a}^{+}=\frac{\partial}{\partial \theta^{-a}}, \quad D^{\hat{+a}}=\frac{\partial}{\partial \theta_{a}^{\hat{\alpha}}},
$$

which reflects the existence of the closed analytic subspace with the double set of Grassmann and harmonic coordinates:

$$
\hat{\zeta}:=\left(x^{(a n) a b}, \theta^{+a}, \theta_{a}^{\hat{+}}, u^{ \pm i}, u_{A}^{\hat{ \pm}}\right) .
$$

Note that one can impose the Grassmann analyticity conditions either separately with respect to $\theta^{-a}$ and $\theta_{a}^{\hat{-}}$, or simultaneously with respect to both these coordinates, without contradiction with the coordinate action of $\mathscr{N}=4$ Poincare supersymmetry in the basis (2.18). The second option corresponds to the minimal-dimension " $1 / 2$ " analytic subspace (2.20), while the first one to the existence of the additional " $3 / 4$ " analytic subspaces $\hat{\zeta}_{I}$ and $\hat{\zeta}_{I I}$ parametrized by the following sets of coordinates

$$
\hat{\zeta}_{I}=\left(\hat{\zeta}, \theta^{-a}\right), \quad \hat{\zeta}_{I I}=\left(\hat{\zeta}, \theta_{a}^{-}\right) .
$$

They are also closed under total $\mathscr{N}=(1,1)$ supersymmetry. While $\hat{\zeta}$ involves eight Grassmann coordinates compared to sixteen such coordinates of the full $\mathscr{N}=(1,1)$ HSS, both $3 / 4$ analytic subspaces contain twelve Grassmann coordinates.

The harmonic derivatives with respect to $u_{A}^{\hat{x}}$ in the analytic basis have the same structure as the $\mathscr{N}=(1,0)$ ones defined earlier, modulo the proper altering of the position of the spinor indices. The same concerns their mutual commutators, their commutation relations with the hat-spinor derivatives, and the mutual anticommutators of the hat-spinor derivatives, taking into account the relation

$$
\partial^{a b}=\frac{1}{2} \varepsilon^{a b c d} \partial_{c d}
$$

To complete this section, we define the integration measures for the full and analytic $\mathscr{N}=(1,0)$ and $\mathscr{N}=(1,1) \mathrm{HSS}$

$$
\begin{aligned}
d Z & =d^{6} x^{(a n)} d u\left(D^{-}\right)^{4}\left(D^{+}\right)^{4}, & d \zeta^{(-4)} & =d^{6} x^{(a n)} d u\left(D^{-}\right)^{4}, \\
d \hat{Z} & =d Z d \hat{u}\left(D^{-}\right)^{4}\left(D^{\hat{+}}\right)^{4}, & d \hat{\zeta}^{(-4, \hat{-} 4)} & =d \zeta^{-4} d \hat{u}\left(D^{\hat{-}}\right)^{4}, \\
d \hat{\zeta}_{I}^{(0, \hat{-} 4)} & =d Z d \hat{u}\left(D^{\hat{-}}\right)^{4}, & d \hat{\zeta}_{I I}^{(-4,0)} & =d \zeta^{(-4)} d \hat{u}\left(D^{\hat{-}}\right)^{4}\left(D^{\hat{+}}\right)^{4}, \\
\left(D^{ \pm}\right)^{4} & =-\frac{1}{24} \varepsilon^{a b c d} D_{a}^{ \pm} D_{b}^{ \pm} D_{c}^{ \pm} D_{d}^{ \pm}, & \left(D^{\hat{ \pm}}\right)^{4} & =-\frac{1}{24} \varepsilon_{a b c d} D^{\hat{ \pm} a} D^{\hat{ \pm} b} D^{\hat{ \pm} c} D^{\hat{ \pm} d} .
\end{aligned}
$$




\section{$3 \mathscr{N}=(1,0)$ supersymmetric Yang-Mills theory}

\subsection{Kinematics}

Now we turn to supersymmetric Yang-Mills (SYM) theory in $6 D, \mathscr{N}=(1,0)$ HSS $[21,22$, $26,32] .{ }^{4}$ First, we introduce the gauge superfield $V^{++}$that covariantizes the harmonic derivative

$$
\nabla^{++}=D^{++}+V^{++} .
$$

The connection $V^{++}$is a G-analytic superfield that transforms as $\delta V^{++}=\nabla^{++} \Lambda$ under gauge transformations with a G-analytic gauge parameter $\Lambda(\zeta)$. The second harmonic derivative $D^{--}$is covariantized by a non-analytic connection $V^{--}$,

$$
\nabla^{--}=D^{--}+V^{--} .
$$

This connection is uniquely determined from the zero-curvature ("harmonic flatness") condition,

$$
\left[\nabla^{++}, \nabla^{--}\right]=D^{0} \Rightarrow D^{++} V^{--}-D^{--} V^{++}+\left[V^{++}, V^{--}\right]=0,
$$

as a series over the connections $V^{++}$given at different harmonic "points":

$$
V^{--}(z, u)=\sum_{n=1}^{\infty}(-1)^{n} \int d u_{1} \ldots d u_{n} \frac{V^{++}\left(z, u_{1}\right) \ldots V^{++}\left(z, u_{n}\right)}{\left(u^{+} u_{1}^{+}\right)\left(u_{1}^{+} u_{2}^{+}\right) \ldots\left(u_{n}^{+} u^{+}\right)},
$$

where $1 /\left(u^{+} u_{1}^{+}\right), \ldots$ are harmonic distributions defined in [20]. It is straightforward to show that $\delta V^{--}=\nabla^{--} \Lambda .{ }^{5}$

Defining

$$
\mathscr{D}_{a}^{-}=D^{-a}+\mathscr{A}_{a}^{-}, \quad \mathscr{D}_{a b}=\partial_{a b}+\mathscr{A}_{a b}
$$

and imposing the commutation relations

$$
\begin{aligned}
{\left[\nabla^{--}, D_{a}^{+}\right] } & =\mathscr{D}_{a}^{-}, \quad\left[\nabla^{++}, \mathscr{D}_{a}^{-}\right]=D_{a}^{+}, \quad\left[\nabla^{++}, D_{a}^{+}\right]=\left[\nabla^{--}, \mathscr{D}_{a}^{-}\right]=0, \\
\left\{D_{a}^{+}, \mathscr{D}_{b}^{-}\right\} & =2 i \mathscr{D}_{a b},
\end{aligned}
$$

we can express the spinor and vector connections in terms of $V^{--}$:

$$
\mathscr{A}_{a}^{-}(V)=-D_{a}^{+} V^{--}, \quad \mathscr{A}_{a b}(V)=\frac{i}{2} D_{a}^{+} D_{b}^{+} V^{--} .
$$

The covariant spinor superfield strength $W^{+a}$ is defined as

$$
\left[D_{a}^{+}, \mathscr{D}_{b c}\right]=\frac{i}{2} \varepsilon_{a b c d} W^{+d}, \quad W^{+a}=-\frac{1}{6} \varepsilon^{a b c d} D_{b}^{+} D_{c}^{+} D_{d}^{+} V^{--} .
$$

For later use, we also define

$$
W^{-a}:=\nabla^{--} W^{+a}, \quad \delta W^{ \pm a}=-\left[\Lambda, W^{ \pm a}\right]
$$

\footnotetext{
${ }^{4}$ See also [33-35].

${ }^{5}$ This and many other properties can be proved using the lemma: $\nabla^{++} F^{-n}=0 \Rightarrow F^{-n}=0$ for $n \geq 1$, where $F^{-n}$ is a harmonic $\mathscr{N}=(1,0)$ superfield.
} 
so that

$$
\left[\mathscr{D}_{a}^{-}, \mathscr{D}_{b c}\right]=\frac{i}{2} \varepsilon_{a b c d} W^{-d} .
$$

The spinor superfield strengths obey the following off-shell relations

$$
\begin{array}{rlrl}
\nabla^{++} W^{+a} & =\nabla^{--} W^{-a}=0, & \nabla^{++} W^{-a} & =W^{+a}, \\
D_{b}^{+} W^{+a} & =\delta_{b}^{a} F^{++}, & F^{++}:=\left(D^{+}\right)^{4} V^{--}=\frac{1}{4} D_{a}^{+} W^{+a}, \\
\nabla^{++} F^{++} & =D_{a}^{+} F^{++}=0 & &
\end{array}
$$

(as well as some other ones derived through acting by $\nabla^{--}$on eqs. (3.11), (3.12)). An important Bianchi identity stemming from (3.8) and (3.10) is

$$
\left[\mathscr{D}_{a b}, \mathscr{D}_{c d}\right]=\frac{1}{4}\left(\varepsilon_{a c d f} \mathscr{D}_{b}^{-} W^{+f}+\varepsilon_{b c d f} D_{a}^{+} W^{-f}\right),
$$

which, in particular, implies

$$
\begin{aligned}
\mathscr{D}_{b}^{-} W^{+b} & =D_{b}^{+} W^{-b}, \\
{\left[\mathscr{D}^{a b}, \mathscr{D}_{c b}\right] } & =\frac{1}{4}\left(D_{c}^{+} W^{-a}-\mathscr{D}_{c}^{-} W^{+a}\right), \\
\mathscr{D}_{a b} W^{+b} & =\frac{i}{8} \mathscr{D}_{a}^{-} D_{c}^{+} W^{+c}, \quad \mathscr{D}_{a b} W^{-b}=-\frac{i}{8} D_{a}^{+} \mathscr{D}_{c}^{-} W^{-c} .
\end{aligned}
$$

\subsection{Dynamics}

The superfield action of $6 D, \mathscr{N}=(1,0)$ SYM theory can be written in the form [22]

$$
S^{\mathrm{SYM}}=\frac{1}{f^{2}} \sum_{n=2}^{\infty} \frac{(-1)^{n}}{n} \operatorname{Tr} \int d^{6} x d^{8} \theta d u_{1} \ldots d u_{n} \frac{V^{++}\left(z, u_{1}\right) \ldots V^{++}\left(z, u_{n}\right)}{\left(u_{1}^{+} u_{2}^{+}\right) \ldots\left(u_{n}^{+} u_{1}^{+}\right)},
$$

where $f$ is a coupling constant with the dimension of inverse mass, so that the relevant component Lagrangian density has the canonical ("engineering") dimension $d=4$. The action (3.17) is invariant under the supergauge transformations of $V^{++}$and yields the equation of motion

$$
F^{++}=\left(D^{+}\right)^{4} V^{--}=0 .
$$

Both these properties can be derived by employing the variation formula

$$
\delta S^{\mathrm{SYM}}=-\frac{1}{f^{2}} \operatorname{Tr} \int d Z \delta V^{++} V^{--}=-\frac{1}{f^{2}} \operatorname{Tr} \int \zeta^{(-4)} \delta V^{++} F^{++} .
$$

Using the representation

$$
F^{++}=\frac{1}{4} D_{a}^{+} W^{+a},
$$

eq. (3.18) can be rewritten in terms of the spinor superfield strength as

$$
D_{a}^{+} W^{+a}=0 \quad \text { or } \quad D_{a}^{+} W^{+b}=0 .
$$


Due to the gauge freedom with the analytic super-parameter $\Lambda$, one can choose the Wess-Zumino gauge for $V^{++}$

$$
\begin{aligned}
V^{++} & =i \theta^{+a} \theta^{+b} A_{a b}+\frac{1}{3} \Psi_{a}^{+3} \lambda^{-a}+\frac{1}{8} \Psi^{+4} \mathscr{D}^{-2}, \\
\lambda^{-a} & =\lambda^{i a}(x) u_{i}^{-}, \quad \mathscr{D}^{-2}=\mathscr{D}^{(i k)}(x) u_{i}^{-} u_{k}^{-},
\end{aligned}
$$

where

$$
\Psi_{d}^{+3}:=\varepsilon_{a b c d} \theta^{+a} \theta^{+b} \theta^{+c}, \quad \Psi^{+4}:=\varepsilon_{a b c d} \theta^{+a} \theta^{+b} \theta^{+c} \theta^{+d} .
$$

The fields entering (3.22) form the component off-shell $6 D, \mathscr{N}=(1,0) \mathrm{SYM}$ multiplet.

Along with $V^{++}$, an important ingredient of the harmonic superspace formulation of $6 D, \mathscr{N}=(1,1)$ SYM theory is the analytic hypermultiplet superfield $q^{+A}(\zeta)$ which also takes values in the adjoint representation of the gauge group and satisfies the reality condition

$$
\widetilde{q^{+A}}=\varepsilon_{A B} q^{+B}
$$

Its action reads

$$
S^{q}=\frac{1}{2 f^{2}} \operatorname{Tr} \int d \zeta^{(-4)} q^{+A} \nabla^{++} q_{A}^{+}
$$

The sum of two actions,

$$
S_{(1,1)}=S^{\mathrm{SYM}}+S_{q}
$$

reveals a hidden $\mathscr{N}=(0,1)$ supersymmetry $[26]$,

$$
\delta_{(0,1)} V^{++}=\epsilon^{+A} q_{A}^{+}, \quad \delta_{(0,1)} q^{+A}=-\left(D^{+}\right)^{4}\left(\epsilon^{-A} V^{--}\right), \quad \epsilon^{ \pm A}:=\epsilon_{a}^{A} \theta^{ \pm a},
$$

where $\epsilon_{a}^{A}$ is the corresponding Grassmann parameter. ${ }^{6}$ So the total action describes $\mathscr{N}=$ $(1,1)$ SYM theory. The equation of motion for the hypermultiplet is

$$
\nabla^{++} q^{+A}=0 .
$$

The equation of motion for $V^{++}$following from the action $S_{(1,1)}$ undergoes the evident modification as compared to (3.18):

$$
F^{++}+\frac{1}{2}\left[q^{+A}, q_{A}^{+}\right]=0 .
$$

On the mass shell, the previously listed Bianchi identities are simplified and some new relations of this kind can be derived. In the limit of vanishing hypermultiplet superfield (this limit will be employed in section 7), the equations of motion (3.21), combined with the relation (3.14), imply

$$
D_{a}^{+} W^{-b}=-\mathscr{D}_{a}^{-} W^{+b}, \quad D_{a}^{+} W^{-a}=\mathscr{D}_{a}^{-} W^{+a}=\mathscr{D}_{a}^{-} W^{-a}=0 .
$$

\footnotetext{
${ }^{6}$ The transformations (3.27) provide an off-shell invariance of (3.26). However, their closure with the manifest $\mathscr{N}=(1,0)$ supersymmetry amounts to the standard relations of $6 D, \mathscr{N}=(1,1)$ Poincaré superalgebra only on shell.
} 
The Bianchi identity (3.16) yields

$$
\mathscr{D}_{a b} W^{ \pm b}=0
$$

or, in an equivalent form,

$$
\mathscr{D}^{a b} W^{ \pm c}+\mathscr{D}^{c a} W^{ \pm b}+\mathscr{D}^{b c} W^{ \pm a}=0 .
$$

The last two identities, together with (3.15), give rise to the equation

$$
\mathscr{D}_{a b} \mathscr{D}^{a b} W^{ \pm c}=-\left[D_{b}^{+} W^{-c}, W^{ \pm b}\right]=\left[\mathscr{D}_{b}^{-} W^{+c}, W^{ \pm b}\right] .
$$

Eqs. (3.31) and (3.33) are none other than the covariantized 6D Dirac and Klein-FockGordon equations for the $\mathscr{N}=(1,0)$ vector multiplet. In the abelian limit they are reduced to

$$
\partial_{a b} W^{ \pm b}=0, \quad \partial_{a b} \partial^{a b} W^{ \pm c}=0
$$

\section{$4 \mathscr{N}=(1,1) \mathrm{SYM}$ in bi-HSS formulation}

When $6 D, \mathscr{N}=(1,1)$ SYM theory is formulated in terms of $\mathscr{N}=(1,1)$ superfields $([17,23]$ and [15]), the constraints of the theory imply equations of motions, i.e. this formulation supplies an example of the on-shell description.

The original $\mathscr{N}=(1,1)$ SYM constraints, before introducing the harmonic coordinates, are written in terms of the gauge-covariantized spinor derivatives,

$$
\nabla_{a}^{i}=\frac{\partial}{\partial \theta_{i}^{a}}-i \theta^{b i} \partial_{a b}+\mathscr{A}_{a}^{i}, \quad \hat{\nabla}^{a A}=\frac{\partial}{\partial \hat{\theta}_{A a}}-i \hat{\theta}_{b}^{A} \partial^{a b}+\hat{\mathscr{A}}^{a A},
$$

where $\mathscr{A}_{a}^{i}$ and $\hat{\mathscr{A}}^{a A}$ are the spinor connections and the convention $\nabla^{a b}=\frac{1}{2} \varepsilon^{a b c d} \nabla_{c d}$ is assumed. The constraints read [17, 23]

$$
\begin{aligned}
& \left\{\nabla_{a}^{(i}, \nabla_{b}^{j)}\right\}=\left\{\hat{\nabla}^{a(A}, \hat{\nabla}^{b B)}\right\}=0, \\
& \left\{\nabla_{a}^{i}, \hat{\nabla}^{b A}\right\}=\delta_{a}^{b} \phi^{i A} .
\end{aligned}
$$

Through the Bianchi identities, eqs. (4.2) and (4.3) imply

$$
\nabla_{a}^{(i} \phi^{j) A}=\hat{\nabla}^{a(A} \phi^{i B)}=0 .
$$

The constraints $(4.2)$ and (4.3) define $6 D, \mathscr{N}=(1,1)$ SYM supersymmetric theory in the standard $\mathscr{N}=(1,1)$ superspace parametrized by the coordinates $\left(x^{a b}, \theta_{i}^{a}, \hat{\theta}_{A a}\right)$. As was already mentioned, they imply the equations of motion for the superfields involved and so put the theory on shell.

The standard steps of the harmonic interpretation of the above constraints can be summarized as: $(i)$ contracting the $\mathrm{SU}(2)$ indices of the spinor derivatives and superfield strengths with the harmonics $u_{i}^{ \pm}, u_{A}^{\hat{y}}$, thereby passing to the harmonic projections of these derivatives; (ii) replacing the property of linearity in harmonics by the property of vanishing of some commutation relations with the harmonic derivatives; (iii) passing to the 
analytic basis and frame, so as to make "short" (i.e. having no connection in this frame) as much spinor derivatives as possible. After these steps the constraints are rewritten as the integrability conditions for some sort of Grassmann analyticity, while the underlying gauge multiplet proves to be carried by the harmonic gauge connections covariantizing flat harmonic derivatives.

This general scheme, being applied to the case at hand, yields the following equivalent set of the constraints [26]

$$
\begin{aligned}
\left\{\nabla_{a}^{+}, \nabla_{b}^{+}\right\} & =\left\{D^{\hat{+} a}, D^{\hat{+} b}\right\}=0, \quad\left\{\nabla_{a}^{+}, D^{\hat{+} b}\right\}=\delta_{a}^{b} \phi^{+\hat{+}}, \\
{\left[\nabla^{++}, \nabla_{a}^{+}\right] } & =\left[\nabla^{\hat{+} \hat{+}}, \nabla_{a}^{+}\right]=\left[\nabla^{++}, D^{\hat{+} a}\right]=\left[\nabla^{\hat{+}}, D^{\hat{+} a}\right]=0, \\
{\left[\nabla^{++}, \nabla^{\hat{+}}\right] } & =0,
\end{aligned}
$$

where we have chosen the "hat-analytic" basis, with a short hat-spinor derivative, $\nabla^{\hat{+} a}=$ $D^{\hat{+} a}=\partial / \partial \theta_{a}^{\hat{-}}$. All other derivatives have non-trivial gauge connections

$$
\nabla_{a}^{+}=D_{a}^{+}+\mathscr{A}_{a}^{+}, \quad \nabla^{++}=D^{++}+\tilde{V}^{++}, \quad \nabla^{\hat{+} \hat{+}}=D^{\hat{+} \hat{+}}+V^{\hat{+} \hat{+}} .
$$

The constraints (4.5) are covariant under the gauge transformations with the $3 / 4$ hatanalytic parameter $\tilde{\Lambda}$ :

$$
\begin{aligned}
& \delta \tilde{V}^{++}=\nabla^{++} \tilde{\Lambda}, \delta V^{\hat{+} \hat{+}}=\nabla^{\hat{+}} \tilde{\Lambda}, \delta \mathscr{A}_{a}^{+}=\nabla_{a}^{+} \tilde{\Lambda}, \quad \delta \phi^{+\hat{+}}=\left[\phi^{+\hat{+}}, \tilde{\Lambda}\right] \\
& D^{\hat{+} a} \tilde{\Lambda}=0 \Rightarrow \tilde{\Lambda}=\tilde{\Lambda}\left(\hat{\zeta}, \theta^{-a}\right)=\tilde{\Lambda}\left(\hat{\zeta}_{I}\right) .
\end{aligned}
$$

Eqs. (4.5) impose non-trivial on-shell conditions on the gauge connections. The notable property of the constraints in the form (4.5) is that the derivative $\nabla_{a}^{+}$cannot be chosen "flat" simultaneously with $\nabla^{\hat{+} a}$ because of their non-vanishing anticommutator.

The full set of the relations solving $(4.5)$ in terms of $\mathscr{N}=(1,0)$ superfields was found in [26]. Below we shall briefly summarize them.

- An important result is that, after imposing the appropriate gauge fixing and due to the vanishing of the commutator between the hatted and unhatted covariant harmonic derivatives, the connections $V^{++}$and $V^{--}$prove to coincide with their $\mathscr{N}=(1,0)$ prototypes,

$$
\tilde{V}^{++}=V^{++}(\zeta), \quad D^{++} V^{--}-D^{--} V^{++}+\left[V^{++}, V^{--}\right]=0 .
$$

- For the $\mathscr{N}=(1,1) \mathrm{SYM}$ superfield strength $\phi^{+\hat{+}}$ the following solution was obtained

$$
\begin{aligned}
\phi^{+\hat{+}}= & q^{+\hat{+}}-\theta_{a}^{\hat{+}} W^{+a}-i \theta_{a}^{\hat{+}} \theta_{b}^{\hat{+}} \mathscr{D}^{a b} q^{+\hat{-}}+\frac{1}{6} \Psi^{\hat{+} 3 d}\left[D_{d}^{+} q^{-\hat{-}}, q^{+\hat{-}}\right] \\
& +\frac{1}{24} \Psi^{\hat{+} 4}\left[q^{+\hat{-}},\left[q^{+\hat{-}}, q^{-\hat{-}}\right]\right],
\end{aligned}
$$

where $\Psi^{\hat{+} 3 d}:=\varepsilon^{a b c d} \theta_{a}^{\hat{+}} \theta_{b}^{\hat{+}} \theta_{c}^{\hat{+}}, \Psi^{\hat{+} 4}:=\varepsilon^{a b c d} \theta_{a}^{\hat{+}} \theta_{b}^{\hat{+}} \theta_{c}^{\hat{+}} \theta_{d}^{\hat{+}}, W^{+a}$ was defined in (3.8), $q^{ \pm \hat{ \pm}}=q^{ \pm A} u_{A}^{\hat{ \pm}}, q^{ \pm \hat{F}}=q^{ \pm A} u_{A}^{\hat{F}}$, and

$$
q^{-A}:=\nabla^{--} q^{+A} .
$$

Recall that $\mathscr{D}^{a b}=\frac{1}{2} \varepsilon^{a b c d} \mathscr{D}_{c d}, \mathscr{D}_{c d}=\partial_{c d}+\mathscr{A}_{c d}$ and the vector $\mathscr{N}=(1,0)$ connection $\mathscr{A}_{c d}$ was defined in (3.7). 
- The final expression for the harmonic connection $V^{\hat{+} \hat{+}}$ is as follows

$$
V^{\hat{+} \hat{+}}=i \theta_{a}^{\hat{+}} \theta_{b}^{\hat{+}} \mathscr{A}^{a b}-\frac{1}{3} \Psi^{\hat{+} 3 a} D_{a}^{+} q^{-\hat{-}}+\frac{1}{8} \Psi^{\hat{+} 4}\left[q^{+\hat{-}}, q^{-\hat{-}}\right] .
$$

As a consequence of the second flatness condition,

$$
\left[\nabla^{\hat{+} \hat{+}}, \nabla^{\hat{\mathcal{A}}}\right]=\hat{D}^{0} \Leftrightarrow D^{\hat{+}+} V^{\hat{\mathcal{A}}}-D^{\hat{\mathcal{A}}} V^{\hat{+} \hat{+}}+\left[V^{\hat{+} \hat{+}}, V^{\hat{-} \hat{-}}\right]=0,
$$

the non-analytic harmonic potential $V^{\hat{-\hat{A}}}$ entering $\nabla^{\hat{\mathcal{A}}}=D^{\hat{\mathcal{A}}}+V^{\hat{\mathcal{A}}}$ can be expressed through $V^{\hat{+} \hat{+}}$.

- The constraints (4.5) accompanied by the suitable gauge choice fix the form of the spinor connection $\mathscr{A}_{a}^{+}$entering $\nabla_{a}^{+}$in (4.6) as

$$
\mathscr{A}_{a}^{+}=-\theta_{a}^{\hat{+}} q^{+\hat{-}}+\theta_{a}^{\hat{-}} \phi^{+\hat{+}} .
$$

- The constraints also imply the equations of motion (3.29), (3.28) for the superfields $V^{++}$and $q^{+A}$. Using these equations of motion together with the explicit formulas quoted above (as well as some remote consequences of them collected in appendix A of [26]), one can check the important properties

$$
\nabla^{++} \phi^{+\hat{+}}=\nabla^{\hat{+} \hat{+}} \phi^{+\hat{+}}=0
$$

which, in fact, directly follow from the constraints (4.5) as the Bianchi identities. For further use, we also define

$$
\phi^{-\hat{+}}:=\nabla^{--} \phi^{+\hat{+}}, \phi^{+\hat{\mathcal{-}}}:=\nabla^{\hat{\mathcal{A}}} \phi^{+\hat{+}}, \phi^{-\hat{\mathcal{A}}}=\nabla^{\hat{\mathcal{A}}} \nabla^{--} \phi^{+\hat{+}} .
$$

It is easy to check that

$$
\nabla^{--} \phi^{-\hat{ \pm}}=\nabla^{++} \phi^{+\hat{-}}=\nabla^{\hat{-} \hat{-}} \phi^{ \pm \hat{-}}=\nabla^{\hat{+} \hat{+}} \phi^{-\hat{+}}=0,
$$

and

$$
\nabla^{++} \phi^{-\hat{ \pm}}=\phi^{+\hat{x}}, \quad \nabla^{\hat{+} \hat{+}} \phi^{ \pm \hat{-}}=\phi^{ \pm \hat{+}} .
$$

- The superfield strength $\phi^{+\hat{+}}$ also satisfies the on-shell analyticity conditions

$$
\nabla_{a}^{+} \phi^{+\hat{+}}=D^{\hat{+} a} \phi^{+\hat{+}}=0
$$

which follow as the Bianchi identities from (4.5) and can be explicitly checked using the relations given above, the $\mathscr{N}=(1,0)$ analyticity of $q^{+A}, D_{a}^{+} q^{+A}=0$, and the $\mathscr{N}=(1,0)$ superfield equations of motion.

- After all gauge-fixings and solving the constraints, the only residual gauge freedom is the $\mathscr{N}=(1,0)$ gauge freedom with the analytic $\mathscr{N}=(1,0)$ gauge parameter, $\tilde{\Lambda}\left(\hat{\zeta}, \theta_{a}^{-}\right) \rightarrow \Lambda(\zeta)$. 
As the last steps in constructing the formalism of the differential superspace geometry of $\mathscr{N}=(1,1)$ SYM theory, one defines, in the standard way, the negatively charged covariant spinor derivatives

$$
\nabla_{a}^{-}:=\left[\nabla^{--}, \nabla_{a}^{+}\right], \quad \nabla^{\hat{-} a}:=\left[\nabla^{\hat{\mathcal{A}}}, D^{\hat{+} a}\right]
$$

and the covariant vector derivatives

$$
\begin{aligned}
& \left\{D^{\hat{+} a}, \nabla^{\hat{-} b}\right\}:=2 i \nabla^{a b}=2 i\left(\partial^{a b}+\mathscr{V}^{a b}\right), \quad \mathscr{V}^{a b}=\frac{i}{2} D^{\hat{+} a} D^{\hat{+} b} V^{\hat{-} \hat{-}}, \\
& \left\{\nabla_{a}^{+}, \nabla_{b}^{-}\right\}:=2 i \nabla_{a b}=2 i\left(\partial_{a b}+\mathscr{V}_{a b}\right), \quad \mathscr{V}_{a b}=\frac{1}{2} \varepsilon_{a b c d} \mathscr{V}^{c d} .
\end{aligned}
$$

Note that the identification of the vector connections obtained in two different ways plays an important role in finding the explicit solution of the constraints (4.5) presented above. Also note that, taking into account the expression (4.12), the spinor derivative $\nabla_{a}^{-}$defined in (4.18) has the very simple form

$$
\nabla_{a}^{-}=\mathscr{D}_{a}^{-}-\theta_{a}^{\hat{+}} q^{-\hat{-}}+\theta_{a}^{-\hat{\phi}} \phi^{-\hat{+}} .
$$

In what follows, we will also use the relations

$$
\begin{array}{rlrl}
\left\{D^{\hat{+} a}, \nabla_{b}^{-}\right\} & =\delta_{b}^{a} \phi^{-\hat{+}}, \quad & \left\{\nabla^{\hat{-a}}, \nabla_{b}^{ \pm}\right\} & =\delta_{b}^{a} \phi^{ \pm \hat{-}}, \\
D^{\hat{+} a} \phi^{-\hat{-}} & =-\nabla^{\hat{-} a} \phi^{-\hat{+}}, & \nabla_{a}^{+} \phi^{-\hat{+}} & =-\nabla_{a}^{-} \phi^{+\hat{+}}, \quad \nabla_{a}^{-} \phi^{-\hat{ \pm}}=\nabla^{\hat{-} a} \phi^{ \pm \hat{-}}=0, \\
D^{\hat{+} a} D^{\hat{+} b} \phi^{-\hat{-}} & =-2 i \nabla^{a b} \phi^{-\hat{+}},
\end{array}
$$

which can be easily deduced from the previous relations and definitions. Some other useful relations following as Bianchi identities from the basic ones read

$$
\begin{aligned}
{\left[D^{\hat{+} a}, \nabla_{b c}\right] } & =\frac{i}{2}\left(\delta_{b}^{a} \nabla_{c}^{-}-\delta_{c}^{a} \nabla_{b}^{-}\right) \phi^{+\hat{+}}, \quad\left[\nabla^{\hat{-} a}, \nabla_{b c}\right]=\frac{i}{2}\left(\delta_{b}^{a} \nabla_{c}^{-}-\delta_{c}^{a} \nabla_{b}^{-}\right) \phi^{+\hat{-}}, \\
{\left[\nabla_{a}^{ \pm}, \nabla^{b c}\right] } & =-\frac{i}{2}\left(\delta_{a}^{b} D^{\hat{+} c}-\delta_{a}^{c} D^{\hat{+} b}\right) \phi^{ \pm \hat{-}} \\
{\left[\nabla_{a b}, \nabla^{c d}\right] } & =\frac{1}{4}\left[\delta_{a}^{c} \nabla_{b}^{-} \nabla^{\hat{-} d}-\delta_{a}^{d} \nabla_{b}^{-} \nabla^{\hat{-} c}-(a \leftrightarrow b)\right] \phi^{+\hat{+}} .
\end{aligned}
$$

Now we are armed with all the necessary ingredients for constructing on-shell invariants of $6 D, \mathscr{N}=(1,1)$ SYM theory in $\mathscr{N}=(1,1)$ harmonic superspace, in the manifestly gauge invariant and $6 D, \mathscr{N}=(1,1)$ supersymmetric fashion.

\section{On-shell $\mathscr{N}=(1,1)$ SYM invariants}

\subsection{Generalities}

The building-blocks of all higher-dimension invariants of $\mathscr{N}=(1,1)$ SYM theory in the bi-harmonic approach are the superfield strength $\phi^{+\hat{+}}$, its various harmonic projections obtained by acting on it by the gauge-covariant harmonic derivatives $\nabla^{ \pm \pm}, \nabla^{\hat{x} \hat{x}}$, as well as the superfields obtained through acting of the spinor and vector gauge-covariant derivatives 
$\nabla_{a}^{ \pm}, D^{\hat{+} a}, \nabla^{\hat{-} a}$ and $\nabla_{a b}$ on the harmonic projections just mentioned. These objects live either on the entire $\mathscr{N}=(1,1)$ bi-HSS or on its analytic subspaces. To ensure gauge invariance, one is led to take traces of the products of such elementary matrix blocks, each block being valued in the adjoint representation of the gauge group algebra (like $\phi^{+\hat{+}}$ ). As shown in [26], given some covariant $\mathscr{N}=(1,1)$ superfield $\Phi(\hat{Z})$, the transformations of hidden $\mathscr{N}=(0,1)$ supersymmetry on the $\mathscr{N}=(1,0)$ superfield constituents of such products, before taking traces, are induced by the following generic transformation of $\Phi(\hat{Z})$

$$
\delta_{(0,1)} \Phi(\hat{Z})=\delta_{\text {stand }} \Phi(\hat{Z})+\left[\delta_{(1)}^{\text {comp }}+\delta_{(2)}^{\text {comp }}\right] \Phi(\hat{Z}) .
$$

Here, $\delta_{\text {stand }}$ is the standard $\mathscr{N}=(0,1)$ superfield transformation in the analytic basis induced by the proper shifts of the superspace coordinates,

$$
\delta_{\text {stand }} \Phi(\hat{Z})=-\left(\epsilon_{a}^{\hat{+}} \frac{\partial}{\partial \theta_{a}^{\hat{+}}}+\epsilon_{a}^{\hat{-}} \frac{\partial}{\partial \theta_{a}^{\hat{\alpha}}}+2 i \epsilon_{a}^{\hat{-}} \theta_{b}^{\hat{+}} \partial^{a b}\right) \Phi(\hat{Z}),
$$

while $\delta_{(1)}^{\text {comp }}$ and $\delta_{(2)}^{\text {comp }}$ are compensating gauge transformations with analytic parameters $\tilde{\Lambda}_{(1)}, \tilde{\Lambda}_{(2)}$ needed to preserve various superfield gauges imposed in the process of solving the constraints $(4.5)$

$$
\tilde{\Lambda}_{(1)}=2 i \epsilon_{a}^{\hat{-}} \theta_{b}^{\hat{+}} \mathscr{A}^{a b}+\text { higher orders in } \theta_{b}^{\hat{+}}, \quad \tilde{\Lambda}_{(2)}=\epsilon^{-B} q_{B}^{+},
$$

where the higher-order $\theta_{a}^{\hat{+}}$ terms in $\tilde{\Lambda}_{(1)}$ are of no interest for us here. Actually, the same formula for variation is applicable not only to gauge-covariant expressions but equally for the various gauge connections. Applying it to the basic entity $\phi^{+\hat{+}}$ defined in (4.9), we find the following $\mathscr{N}=(0,1)$ transformation of the basic $\mathscr{N}=(1,0)$ superfield objects

$$
\delta_{(0,1)} q^{+A}=\epsilon_{b}^{A} W^{+b}-\left[\epsilon^{-B} q_{B}^{+}, q^{+A}\right], \quad \delta_{(0,1)} W^{+a}=-2 i \epsilon_{b}^{A} \mathscr{D}^{a b} q_{A}^{+}-\left[\epsilon^{-B} q_{B}^{+}, W^{+a}\right] .
$$

These transformations could be as well directly derived from (3.27) by imposing the equations of motion (3.28), (3.29). For $q^{-A}=\nabla^{--} q^{+A}$ and $W^{-b}=\nabla^{--} W^{+b}$ one obtains the same transformation rules.

An important point is that one can omit all the gauge transformation parts in (5.1), when constructing the higher-order invariants, since the superfields like $\Phi(\hat{Z})$ always appear under the trace. So one can construct actual $\mathscr{N}=(1,1)$ SYM invariants by integrating $\Phi(\hat{Z})$ over the appropriate invariant subspaces of $\mathscr{N}=(1,1)$ bi-HSS.

We start by recalling some invariants obtained in [26]. In what follows, the dimension d of diverse invariants is understood as the canonical dimension (in the mass units) of their component Lagrangian. So the dimension of the "microscopic" action $S_{(1,1)}$ is $\mathbf{d}=\mathbf{4}$. Note that it makes no sense to ask whether this simplest invariant can be represented as an integral over $\mathscr{N}=(1,1)$ superspace since an essential feature of our formalism is that it is on-shell. The relevant $\mathscr{N}=(1,0)$ superfields are assumed to satisfy the equations of motion (3.28), (3.29) following just from $S_{(1,1)}$, so the latter vanishes on the shell of these equations. It will be useful to list the dimensions of the basic building blocks:

$$
\left[\nabla_{a}^{ \pm}\right]=\left[\nabla^{\hat{-} a}\right]=\left[D^{\hat{+} a}\right]=1 / 2, \quad\left[\nabla^{a b}\right]=1, \quad\left[\nabla^{ \pm \pm}\right]=\left[\nabla^{\hat{ \pm} \hat{ \pm}}\right]=0, \quad\left[\phi^{+\hat{+}}\right]=1 .
$$


Also, we list the dimensions of various $\mathscr{N}=(1,0)$ and $\mathscr{N}=(1,1)$ superspace integration measures defined in (2.22)-(2.24):

$$
\begin{aligned}
{[d Z] } & =-2, \quad\left[d \hat{\zeta}^{(-4)}\right]=-4, \\
{[d \hat{Z}] } & =2, \quad\left[d \hat{\zeta}^{(-4, \hat{-} 4)}\right]=-2, \quad\left[d \hat{\zeta}_{I}^{(0, \hat{-} 4)}\right]=\left[d \hat{\zeta}_{I I}^{(-4,0)}\right]=0 .
\end{aligned}
$$

It is worth to have in mind that, due to the presence of trace in the possible invariants, it is legal to integrate by parts with respect to the harmonic, spinor and $x$-derivatives, e.g., $\operatorname{Tr} \nabla^{++} \Omega=D^{++} \operatorname{Tr} \Omega$, etc. The same is true for the invariants the Lagrangian densities of which are representable as products of few traces. For what follows, it will be useful to give the general definition of various invariants as integrals over the full bi-harmonic superspace and its various subspaces

$$
\begin{array}{rlrl}
S & :=\int d \hat{Z} L, & \mathscr{S} & :=\int d \hat{\zeta}^{(-4, \hat{-}-4)} \mathscr{L}^{(+4, \hat{+} 4)}, \\
\mathscr{S}_{I} & :=\int d \hat{\zeta}_{I}^{(0, \hat{-} 4)} \mathscr{L}_{I}^{(0, \hat{+} 4)}, & \mathscr{S}_{I I}:=\int d \hat{\zeta}_{I I}^{(-4,0)} \mathscr{L}_{I I}^{(+4,0)} .
\end{array}
$$

\subsection{Examples}

As promised, we start with recalling the results of [26].

$\mathbf{d}=4$. For completeness, we will start from the case with the canonical dimension of the microscopic $\mathscr{N}=(1,1) \mathrm{SYM}$ action. In this case the dimension of possible extra invariant is $\mathbf{d}=-\mathbf{2}$, so we obtain

$$
[L]=-4, \quad\left[\mathscr{L}^{(+4, \hat{+} 4)}\right]=0, \quad\left[\mathscr{L}_{I}^{(0, \hat{+} 4)}\right]=\left[\mathscr{L}_{I I}^{(+4,0)}\right]=-2 .
$$

It is impossible to construct such Lagrangian densities from the basic building blocks mentioned in the beginning of this section, because all of them have the strictly positive dimensions.

$\mathbf{d}=\mathbf{6}$. In this case the possible invariants should be dimensionless, so we have

$$
[L]=-2, \quad\left[\mathscr{L}^{(+4, \hat{+} 4)}\right]=2, \quad\left[\mathscr{L}_{I}^{(0, \hat{+} 4)}\right]=\left[\mathscr{L}_{I I}^{(+4,0)}\right]=0 .
$$

All invariants except the second one are ruled out by the dimensionality reasoning, like in the $\mathbf{d}=\mathbf{4}$ case. It is easy to be convinced that no analytic bi-harmonic densities of the dimension 2 and the charges $(+4, \hat{+} 4)$ can be constructed from the basic "bricks". So there exist no on-shell $\mathscr{N}=(1,1)$ invariants with the component Lagrangian density of the canonical dimension 6 . This amounts to the one-loop finiteness of $6 D, \mathscr{N}=(1,1) \mathrm{SYM}$ theory.

$\mathbf{d}=\mathbf{8}$. The relevant invariants should have the dimension $\mathbf{d}=\mathbf{2}$, that implies

$$
[L]=0, \quad\left[\mathscr{L}^{(+4, \hat{+} 4)}\right]=4, \quad\left[\mathscr{L}_{I}^{(0, \hat{+} 4)}\right]=\left[\mathscr{L}_{I I}^{(+4,0)}\right]=2 .
$$

Like in the previous case, one can rule out the last two options since no hat-analytic or analytic densities of the dimension 2 and the charges +4 or $\hat{+} 4$ can be constructed out of 
the basic blocks. The first option is dismissed on the dimensionality grounds, so we are left with the second option. The corresponding superfield single-trace Lagrangian is specified up to an overall constant, so that

$$
\mathscr{S}_{(8)}^{(1)} \sim \operatorname{Tr} \int d \hat{\zeta}^{(-4, \hat{-} 4)}\left(\phi^{+\hat{+}}\right)^{4} .
$$

Note that the Lagrangian in (5.12) is analytic only on shell since the first condition in (4.17) is satisfied with taking into account the $\mathscr{N}=(1,0)$ superfield equations of motion. This means that both manifest and hidden supersymmetries of (5.12) are fulfilled on shell. The same property is shared by the double-trace invariant

$$
\mathscr{S}_{(8)}^{(2)} \sim \int d \hat{\zeta}^{(-4, \hat{-} 4)} \operatorname{Tr}\left(\phi^{+\hat{+}}\right)^{2} \operatorname{Tr}\left(\phi^{+\hat{+}}\right)^{2} .
$$

Since the $6 D, \mathscr{N}=(1,0)$ HSS supergraph techniques [29-31] should yield expressions with off-shell $\mathscr{N}=(1,0)$ supersymmetry, the absence of such invariants indicates the two-loop finiteness of $6 D, \mathscr{N}=(1,1)$ SYM theory. ${ }^{7}$

$\mathbf{d}=\mathbf{1 0}$. The dimension of the relevant invariant is $\mathbf{d}=\mathbf{4}$, so the dimensions of the superfield Lagrangian densities are

$$
[L]=2, \quad\left[\mathscr{L}^{(+4, \hat{+} 4)}\right]=6, \quad\left[\mathscr{L}_{I}^{(0, \hat{+} 4)}\right]=\left[\mathscr{L}_{I I}^{(+4,0)}\right]=4 .
$$

A simple analysis shows that no proper double-analytic expression of the dimension 6 with the charges $(+4, \hat{+} 4)$ exists, even if the equations of motion are assumed. At the same time, the first and last invariants can be easily constructed:

$$
S_{(10)}=\operatorname{Tr} \int d \hat{Z} \phi^{+\hat{+}} \phi^{-\hat{-}}
$$

(another admissible invariant $\sim \phi^{+\hat{-}} \phi^{-\hat{+}}$ is reduced to (5.15) after integration by parts). A single-trace hat-analytic invariant is given by the expression

$$
\mathscr{S}_{I(10)}:=\operatorname{Tr} \int d \hat{\zeta}_{I}^{(0, \hat{-} 4)}\left(\phi^{+\hat{+}}\right)^{2}\left(\phi^{-\hat{+}}\right)^{2} .
$$

It is interesting that both these invariants can be reduced to each other. Representing

$$
d \hat{Z}=d \hat{\zeta}_{I}^{(0, \hat{-} 4)}\left(D^{\hat{+}}\right)^{4}
$$

and taking into account that the factor $\left(D^{\hat{+}}\right)^{4}$ in $(5.15)$ acts only on $\phi^{-\hat{-}}$ in view of the hat-analyticity of $\phi^{+\hat{+}}$, it is rather straightforward to find, using, in particular, the relations $(4.24)$, that

$$
\left(D^{\hat{+}}\right)^{4} \phi^{-\hat{-}}=-\left[\left[\phi^{+\hat{+}}, \phi^{-\hat{+}}\right], \phi^{-\hat{+}}\right] .
$$

Hence,

$$
S_{(10)}=\operatorname{Tr} \int d \hat{\zeta}_{I}^{(0, \hat{-} 4)}\left[\phi^{+\hat{+}}, \phi^{-\hat{+}}\right]\left[\phi^{+\hat{+}}, \phi^{-\hat{+}}\right]
$$

\footnotetext{
${ }^{7}$ The explicit quantum harmonic superfield calculations confirming this statement were performed in $[36,37]$.
} 
On the other hand, using the integration by parts with respect to $\nabla^{\hat{+} \hat{+}}$, one can show that

$$
S_{(10)}=-6 \operatorname{Tr} \int d \hat{\zeta}_{I}^{(0, \hat{-} 4)}\left(\phi^{+\hat{+}}\right)^{2}\left(\phi^{-\hat{+}}\right)^{2} .
$$

So,

$$
S_{(10)}=-6 \mathscr{S}_{I(10)} .
$$

Quite analogously, representing $d \hat{Z}=d \hat{\zeta}_{I I}^{(-4,0)}\left(D^{+}\right)^{4}$ and taking advantage of the property that $D_{a}^{+} \rightarrow \nabla_{a}^{+}$under trace, one can show that

$$
S_{(10)}=\operatorname{Tr} \int d \hat{\zeta}_{I I}^{(-4,0)}\left[\phi^{+\hat{+}}, \phi^{+\hat{-}}\right]\left[\phi^{+\hat{+}}, \phi^{+\hat{-}}\right]=-6 \operatorname{Tr} \int d \hat{\zeta}_{I I}^{(-4,0)}\left(\phi^{+\hat{+}}\right)^{2}\left(\phi^{+\hat{-}}\right)^{2} .
$$

Note an essential difference between the representations (5.20) and (5.22). When passing from $(5.15)$ to $(5.20)$, no use of the $\mathscr{N}=(1,0)$ superfield equations was made, so $\mathscr{N}=(1,0)$ supersymmetry can be still treated as an off-shell supersymmetry, the equations of motion are needed only for demonstrating the hidden $\mathscr{N}=(0,1)$ supersymmetry. The Lagrangian in (5.20) is manifestly hat-analytic since the superfield strengths $\phi^{ \pm \hat{+}}$ do not depend on $\theta_{a}^{-}$. On the other hand, when passing to the representation (5.22), it is imperative to use the equations of motion, because, e.g., the property $\nabla_{a}^{+} \phi^{+\hat{+}}=0$ is valid only on shell. So the Lagrangian in $(5.22)$ is $\mathscr{N}=(1,0)$ analytic only on shell, and $\mathscr{N}=(1,0)$ supersymmetry is valid also on shell, like in the case of $\mathbf{d}=\mathbf{8}$ invariant (5.12). In other words, the equivalency between the initial full bi-harmonic superspace single-trace invariant (5.15) and the invariant (5.20) is valid only on shell. Thus (5.20) provides another example of $\mathscr{N}=(1,1)$ invariants in which both $\mathscr{N}=(1,0)$ and $\mathscr{N}=(0,1)$ supersymmetries are on shell. This peculiarity was not noticed in [26].

As for the double-trace invariants of the dimension $\mathbf{d}=\mathbf{1 0}$, they can be defined only in the $3 / 4$ analytic superspaces,

$$
\begin{aligned}
\mathscr{S}_{(10) I}^{(2)} \sim \int d \hat{\zeta}_{I}^{(0, \hat{-} 4)} \operatorname{Tr}\left(\phi^{+\hat{+}} \phi^{-\hat{+}}\right) \operatorname{Tr}\left(\phi^{+\hat{+}} \phi^{-\hat{+}}\right), \\
\mathscr{S}_{(10) I I}^{(2)} \sim \int d \hat{\zeta}_{I I}^{(-4,0)} \operatorname{Tr}\left(\phi^{+\hat{+}} \phi^{+\hat{-}}\right) \operatorname{Tr}\left(\phi^{+\hat{+}} \phi^{+\hat{-}}\right) .
\end{aligned}
$$

Like in the single-trace case, the Lagrangian density in (5.24) is analytic only on shell, and the same is true for the realization of $\mathscr{N}=(1,0)$ supersymmetry in it. So (5.24) cannot appear as a three-loop divergence in the explicitly $\mathscr{N}=(1,0)$ off-shell supersymmetric perturbation calculations. However, this noticeable property does not fully explain the absence of the double-trace counterterms in the amplitude calculations for $\mathscr{N}=(1,1)$ SYM [8-13], since the alternative double-trace invariant (5.23) is off-shell $\mathscr{N}=(1,0)$ supersymmetric and some extra reasoning is yet needed to rule out it [16].

\section{Invariants of dimension $d=12$}

In this case the relevant component actions should have the canonical dimension $\mathbf{d}=\mathbf{6}$. Respectively, for dimensions of various superfield Lagrangians defined in (5.8) we obtain

$$
[L]=4, \quad\left[\mathscr{L}^{(+4, \hat{+} 4)}\right]=8, \quad\left[\mathscr{L}_{I}^{(0, \hat{+} 4)}\right]=\left[\mathscr{L}_{I I}^{(+4,0)}\right]=6 .
$$


First of all, it is important to realize that it is impossible to construct, out of the elementary "bricks" $\phi^{ \pm \hat{x}}, \phi^{\mp \hat{x}}$ and those obtained from them through action of the covariant differential operators $\nabla_{a}^{ \pm}, D^{\hat{+} a}, \nabla^{\hat{-} a}$ and $\nabla_{a b}$, the gauge invariant and manifestly analytic objects possessing the charges $(+4, \hat{+} 4),(+4,0)$ or $(0, \hat{+} 4)$ and canonical dimensions indicated in (6.1). ${ }^{8}$ So we are left with the chargeless general bi-HSS superfield densities $L$ of the dimension 4 as the only candidates for the invariants we are interested in.

\subsection{Invariants without derivatives}

We start with the invariants containing only superfield strengths $\phi^{ \pm \hat{x}}, \phi^{\mp \hat{x}}$. One can construct ten chargeless superfield invariants of the dimension 4 from these quantities

$$
\begin{array}{ll}
J_{1}=\operatorname{Tr} \phi^{+\hat{+}} \phi^{+\hat{+}} \phi^{-\hat{-}} \phi^{-\hat{-}}, & J_{2}=\operatorname{Tr} \phi^{+\hat{+}} \phi^{-\hat{-}} \phi^{+\hat{+}} \phi^{-\hat{-}}, \\
J_{3}=\operatorname{Tr} \phi^{+\hat{-}} \phi^{+\hat{-}} \phi^{-\hat{+}} \phi^{-\hat{+}}, & J_{4}=\operatorname{Tr} \phi^{+\hat{-}} \phi^{-\hat{+}} \phi^{+\hat{-}} \phi^{-\hat{+}}, \\
I_{1}=\operatorname{Tr} \phi^{+\hat{+}} \phi^{+\hat{-}} \phi^{-\hat{+}} \phi^{-\hat{-}}, & I_{2}=\operatorname{Tr} \phi^{+\hat{+}} \phi^{-\hat{+}} \phi^{-\hat{-}} \phi^{+\hat{-}}, \\
I_{3}=\operatorname{Tr} \phi^{+\hat{+}} \phi^{-\hat{-}} \phi^{+\hat{-}} \phi^{-\hat{+}}, & I_{4}=\operatorname{Tr} \phi^{+\hat{+}} \phi^{-\hat{-}} \phi^{-\hat{+}} \phi^{+\hat{-}}, \\
I_{5}=\operatorname{Tr} \phi^{+\hat{+}} \phi^{+\hat{-}} \phi^{-\hat{-}} \phi^{-\hat{+}}, & I_{6}=\operatorname{Tr} \phi^{+\hat{+}} \phi^{-\hat{+}} \phi^{+\hat{-}} \phi^{-\hat{-}} .
\end{array}
$$

Some of them are related via the integration by parts with respect to harmonic derivatives. To extract the irreducible set, one needs to take off some derivatives with "hat" and/or without "hats" from some superfield factors in (6.2) and (6.3) and place them on the other factors in the same expression, using the relations (4.14), (4.16) and the on-shell harmonic constraints (4.13), (4.15). This can be done in a few equivalent ways. It will be convenient for us to consecutively take off the derivatives $\nabla^{--}, \nabla^{\hat{-1}}$ from all negatively charged factors in the densities $J_{1}, \ldots, J_{4}$. For instance, representing the third factor $\phi^{-\hat{-}}$ in $J_{1}$ under trace as $\phi^{-\hat{-}}=\nabla^{--} \phi^{+\hat{-}}=\nabla^{\hat{-} \hat{-}} \phi^{-\hat{+}}$ and integrating by parts with respect to $\nabla^{--}$and $\nabla^{\hat{-}} \hat{-}$, we obtain, respectively,

$$
\begin{aligned}
& J_{1} \simeq-\operatorname{Tr} \phi^{-\hat{+}} \phi^{+\hat{+}} \phi^{+\hat{-}} \phi^{-\hat{-}}-\operatorname{Tr} \phi^{+\hat{+}} \phi^{-\hat{+}} \phi^{+\hat{-}} \phi^{-\hat{-}}=-I_{5}-I_{6}, \\
& J_{1} \simeq-\operatorname{Tr} \phi^{+\hat{-}} \phi^{+\hat{+}} \phi^{-\hat{+}} \phi^{-\hat{-}}-\operatorname{Tr} \phi^{+\hat{+}} \phi^{+\hat{-}} \phi^{-\hat{+}} \phi^{-\hat{-}}=-I_{2}-I_{1},
\end{aligned}
$$

etc., where $\simeq$ means "up to a total harmonic derivative". In this way we obtain the following set of linear equations (we replace $\simeq$ by $=$ for brevity)

$$
\begin{array}{llll}
J_{1}+I_{5}+I_{6}=0, & J_{1}+I_{2}+I_{1}=0, & J_{1}+I_{3}+I_{2}=0, & J_{1}+I_{4}+I_{5}=0, \\
J_{2}+I_{4}+I_{1}=0, & J_{2}+I_{3}+I_{6}=0, & & \\
J_{3}+I_{2}+I_{6}=0, & J_{3}+I_{3}+I_{5}=0, & J_{3}+I_{1}+I_{5}=0, & J_{3}+I_{2}+I_{4}=0, \\
J_{4}+I_{1}+I_{4}=0, & J_{4}+I_{3}+I_{6}=0 . & &
\end{array}
$$

It is direct to check that applying the same procedure to the densities $I_{1}, \ldots, I_{6}$ produces no new relations. Moreover, the system (6.5)-(6.8) is underdetermined and in fact amounts

\footnotetext{
${ }^{8}$ In principle, some Chern-Simons type not manifestly gauge-invariant densities with these charge and dimension assignments could be imagined (see, e.g. [38-40]); however, here we do not consider such possibilities.
} 
to eight independent equations for ten unknowns. As a result, we are left with the two independent densities. As such it is convenient to choose $J_{1}$ and $J_{2}$ :

$$
I_{1}=I_{3}=I_{4}=I_{6}=-\frac{1}{2} J_{2}, \quad I_{2}=I_{5}=\frac{1}{2} J_{2}-J_{1}, \quad J_{3}=J_{1}, J_{4}=J_{2},
$$

where it is assumed as before that the equalities are valid up to total $\nabla^{--}$or $\nabla^{\hat{-}} \hat{-}$ derivatives.

So in dimension $\mathbf{d}=\mathbf{1 2}$ we have two independent single-trace superfield invariants without derivatives,

$$
S_{(12) I}^{(1)} \sim \operatorname{Tr} \int d \hat{Z} \phi^{+\hat{+}} \phi^{+\hat{+}} \phi^{-\hat{-}} \phi^{-\hat{-}}, \quad S_{(12) I I}^{(1)} \sim \operatorname{Tr} \int d \hat{Z} \phi^{+\hat{+}} \phi^{-\hat{-}} \phi^{+\hat{+}} \phi^{-\hat{-}} .
$$

Analogously, one can define two independent double-trace invariants of the same type:

$$
\begin{aligned}
S_{(12) I}^{(2)} & \sim \int d \hat{Z} \operatorname{Tr}\left(\phi^{+\hat{+}} \phi^{+\hat{+}}\right) \operatorname{Tr}\left(\phi^{-\hat{-}} \phi^{-\hat{-}}\right), \\
S_{(12) I I}^{(2)} & \sim \int d \hat{Z} \operatorname{Tr}\left(\phi^{+\hat{+}} \phi^{-\hat{-}}\right) \operatorname{Tr}\left(\phi^{+\hat{+}} \phi^{-\hat{-}}\right) .
\end{aligned}
$$

\subsection{Invariants with derivatives}

Next we consider possible superfield densities containing spinor and/or vector covariant derivatives. The only possibilities to ensure the total dimension four are the densities with three covariant strengths and two spinor derivatives, or densities with two strengths and either two vector, or four spinor, or two spinor and one vector derivatives properly distributed among these strengths. It is clear that the double-trace invariants of this kind do not exist in view of the tracelessness of the gauge algebra generators.

We start with the three-strength case. Taking into account the on-shell analyticities of various harmonic projections of the superfield strength and the possibility to integrate by parts under the trace, we can construct two options

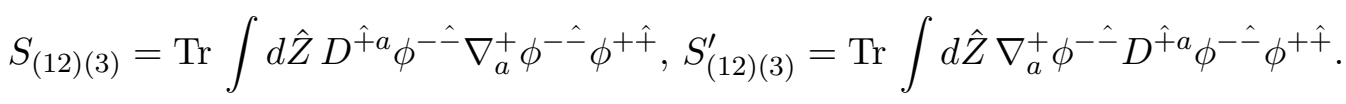

Integrating by parts with respect to $D^{\hat{+} a}$ in the first expression and using $\left\{\nabla_{a}^{+}, D^{\hat{+} a}\right\}=$ $4 \phi^{+\hat{+}}$, we obtain

$$
\begin{aligned}
& \operatorname{Tr} D^{\hat{+} a} \phi^{-\hat{-}} \nabla_{a}^{+} \phi^{-\hat{-}} \phi^{+\hat{+}} \simeq-\operatorname{Tr} \phi^{-\hat{-}} D^{\hat{+} a} \nabla_{a}^{+} \phi^{-\hat{-}} \phi^{+\hat{+}} \\
& =\operatorname{Tr} \phi^{-\hat{-}} \nabla_{a}^{+} D^{\hat{+} a} \phi^{-\hat{-}} \phi^{+\hat{+}}-4 \operatorname{Tr} \phi^{-\hat{-}}\left[\phi^{+\hat{+}}, \phi^{-\hat{-}}\right] \phi^{+\hat{+}} \\
& \simeq-\operatorname{Tr} \nabla_{a}^{+} \phi^{-\hat{-}} D^{\hat{+} a} \phi^{-\hat{-}} \phi^{+\hat{+}}+4 \operatorname{Tr}\left(\phi^{+\hat{+}} \phi^{+\hat{+}} \phi^{-\hat{-}} \phi^{-\hat{-}}-\phi^{+\hat{+}} \phi^{-\hat{-}} \phi^{+\hat{+}} \phi^{-\hat{-}}\right),
\end{aligned}
$$

whence we conclude that $S_{(12)(3)}=-S_{(12)(3)}^{\prime}$ up to the difference of the previous invariants $\sim\left[S_{(12) I}^{(1)}-S_{(12) I I}^{(1)}\right]$. So we are left with only one independent invariant of the type (6.12).

For constructing densities with two superfield strengths there are much more opportunities, but, once again, they are reduced, modulo the invariants already presented, to the following single invariant

$$
S_{(12)(2)} \sim \operatorname{Tr} \int d \hat{Z} \nabla_{a b} \phi^{-\hat{-}} \nabla^{a b} \phi^{+\hat{+}} .
$$


As an example of such a reduction, consider, e.g., the density

$$
\operatorname{Tr} \nabla_{a}^{+} \phi^{-\hat{-}} \nabla_{b}^{-} \nabla^{a b} \phi^{+\hat{+}}
$$

An evident chain of transformations involving integrations by parts, yields

$$
\begin{aligned}
\operatorname{Tr} \nabla_{a}^{+} \phi^{-\hat{-}} \nabla_{b}^{-} \nabla^{a b} \phi^{+\hat{+}} \simeq & -\operatorname{Tr} \phi^{-\hat{-}} \nabla_{a}^{+} \nabla_{b}^{-} \nabla^{a b} \phi^{+\hat{+}}=\operatorname{Tr} \phi^{-\hat{-}} \nabla_{b}^{-} \nabla_{a}^{+} \nabla^{a b} \phi^{+\hat{+}} \\
& -2 i \operatorname{Tr} \phi^{-\hat{-}} \nabla_{a b} \nabla^{a b} \phi^{+\hat{+}} \simeq 2 i \operatorname{Tr} \nabla_{a b} \phi^{-\hat{-}} \nabla^{a b} \phi^{+\hat{+}}
\end{aligned}
$$

where we made use of the relation $\nabla_{b}^{-} \phi^{-\hat{-}}=0$. In a similar way one can handle the density

$$
\operatorname{Tr} D^{\hat{+} a} \phi^{-\hat{-}} \nabla^{\hat{-b}} \nabla_{a b} \phi^{+\hat{+}} .
$$

The density

$$
\operatorname{Tr} \nabla_{a}^{+} \phi^{-\hat{-}} \nabla^{a b} \nabla_{b}^{-} \phi^{+\hat{+}}
$$

can be reduced to (6.15) by using the commutation relations (4.25),

$$
\begin{aligned}
\operatorname{Tr} & \nabla_{a}^{+} \phi^{-\hat{-}} \nabla^{a b} \nabla_{b}^{-} \phi^{+\hat{+}}=\operatorname{Tr} \nabla_{a}^{+} \phi^{-\hat{-}} \nabla_{b}^{-} \nabla^{a b} \phi^{+\hat{+}}-\frac{3 i}{2} \operatorname{Tr} \nabla_{a}^{+} \phi^{-\hat{-}}\left[D^{\hat{+} a} \phi^{-\hat{-}}, \phi^{+\hat{+}}\right] \\
& \simeq \operatorname{Tr} \nabla_{a}^{+} \phi^{-\hat{-}} \nabla_{b}^{-} \nabla^{a b} \phi^{+\hat{+}}-6 i\left(\phi^{+\hat{+}} \phi^{+\hat{+}} \phi^{-\hat{-}} \phi^{-\hat{-}}-\phi^{+\hat{+}} \phi^{-\hat{-}} \phi^{+\hat{+}} \phi^{-\hat{-}}\right)
\end{aligned}
$$

where we made use of the relation (6.13).

Two more examples are the following 'trial' densities

$$
U_{I}=\varepsilon^{a b c d} \operatorname{Tr} \nabla_{a}^{+} \nabla_{b}^{+} \phi^{-\hat{+}} \nabla_{c}^{-} \nabla_{d}^{-} \phi^{+\hat{-}}, \quad U_{I I}=\nabla_{a}^{+} \nabla_{b}^{+} \phi^{-\hat{-}} D^{\hat{+} a} D^{\hat{+} b} \phi^{-\hat{-}} .
$$

Using the relations (4.23), it is easy to reduce them to the expressions

$$
U_{I}=-8 \operatorname{Tr} \nabla_{a b} \phi^{+\hat{+}} \nabla^{a b} \phi^{-\hat{-}}, \quad U_{I I}=-4 \nabla_{a b} \phi^{+\hat{-}} \nabla^{a b} \phi^{-\hat{+}},
$$

which are related to each other via integration by parts with respect to the harmonic derivative $\nabla^{++}$. All other possible expressions, e.g., $\sim \varepsilon_{a b c d} \operatorname{Tr} D^{\hat{+} a} D^{\hat{+} b} \phi^{-\hat{-}} \nabla^{\hat{-} c} \nabla^{\hat{-} d} \phi^{+\hat{+}}$, can be transformed in a similar manner.

For reader's convenience, we finish this section by listing the complete set of independent $\mathscr{N}=(1,1)$ superfield invariants corresponding to the component Lagrangian densities of the canonical dimension $\mathbf{d}=\mathbf{1 2}$.

\section{Single-trace invariants:}

$$
\begin{aligned}
S_{(12) I}^{(1)} & \sim \operatorname{Tr} \int d \hat{Z} \phi^{+\hat{+}} \phi^{+\hat{+}} \phi^{-\hat{-}} \phi^{-\hat{-}}, \quad S_{(12) I I}^{(1)} \sim \operatorname{Tr} \int d \hat{Z} \phi^{+\hat{+}} \phi^{-\hat{-}} \phi^{+\hat{+}} \phi^{-\hat{-}} \\
S_{(12)(3)}^{(1)} & \sim \operatorname{Tr} \int d \hat{Z} D^{\hat{+} a} \phi^{-\hat{-}} \nabla_{a}^{+} \phi^{-\hat{-}} \phi^{+\hat{+}} \\
S_{(12)(2)}^{(1)} & \sim \operatorname{Tr} \int d \hat{Z} \nabla_{a b} \phi^{-\hat{-}} \nabla^{a b} \phi^{+\hat{+}} .
\end{aligned}
$$




\section{Double-trace invariants:}

$$
\begin{aligned}
S_{(12) I}^{(2)} & \sim \int d \hat{Z} \operatorname{Tr}\left(\phi^{+\hat{+}} \phi^{+\hat{+}}\right) \operatorname{Tr}\left(\phi^{-\hat{-}} \phi^{-\hat{-}}\right), \\
S_{(12) I I}^{(2)} & \sim \int d \hat{Z} \operatorname{Tr}\left(\phi^{+\hat{+}} \phi^{-\hat{-}}\right) \operatorname{Tr}\left(\phi^{+\hat{+}} \phi^{-\hat{-}}\right) .
\end{aligned}
$$

It is worth to point out that, as distinct from the $\mathbf{d}=\mathbf{1 0}$ single-trace invariant, the $\mathbf{d}=\mathbf{1 2}$ ones cannot be equivalently rewritten as integrals of the manifestly analytic Lagrangian densities over the appropriate analytic subspaces. It would be interesting to understand possible implications of this property for the structure of quantum corrections in $\mathscr{N}=(1,1) \mathrm{SYM}$ theory. Anyway, in all these invariants $\mathscr{N}=(1,0)$ supersymmetry can be treated as an off-shell one and all of them could in principle appear as divergences in the four-loop order of $6 D, \mathscr{N}=(1,0)$ HSS superfield perturbation calculations. Note that in the abelian case all invariants without derivatives become identical to each other.

\section{Passing to $\mathscr{N}=(1,0)$ superfields}

Here we consider the $\mathscr{N}=(1,0)$ superfield form of some invariants listed in the previous sections. For simplicity, we will confine our attention to the contributions of the $\mathscr{N}=(1,0)$ gauge superfield and ignore the hypermultiplet pieces. So our input for the basic $\mathscr{N}=(1,1)$ superfields defined in (4.9), (4.10) will be the following

$$
\phi^{+\hat{+}} \rightarrow-\theta_{a}^{\hat{+}} W^{+a}, \quad V^{\hat{+} \hat{+}} \rightarrow i \theta_{a}^{\hat{+}} \theta_{b}^{\hat{+}} \mathscr{A}^{a b} .
$$

We also obtain

$$
\phi^{-\hat{+}}=\nabla^{--} \phi^{+\hat{+}} \rightarrow-\theta_{a}^{\hat{+}} W^{-a} .
$$

To compute the remaining $\mathscr{N}=(1,1)$ SYM quantities, we need the explicit expression for $V^{\hat{-}} \hat{-}$ which is defined by the hat-flatness condition (4.11). It is easy to see from the latter that the $\theta_{a}^{\hat{-}}$ expansion of $V^{\hat{-} \hat{-}}$ starts with the second-order terms. So we parametrize $V^{\hat{-} \hat{-}}$ as

$$
V^{\hat{-} \hat{-}}=i \theta_{a}^{\hat{-}} \theta_{b}^{\hat{-}} v^{a b}+\Psi^{\hat{-} 3 d} v_{d}^{\hat{+}}+\Psi^{\hat{-} 4} v^{\hat{+} 2},
$$

where the coefficients are functions of $\left(\theta_{a}^{\hat{+}}, u^{\hat{ \pm A}}\right)$ and of the $\mathscr{N}=(1,0)$ coordinates $Z=$ $\left(x^{a b}, \theta^{ \pm a}, u^{ \pm i}\right)$. From (4.11) we obtain the following equations for these coefficients

$$
\begin{array}{rlrl}
\theta_{a}^{\hat{+}}\left(\mathscr{A}^{a b}-v^{a b}\right) & =0, & \nabla^{\hat{+} \hat{+}} v^{a b}-3 i \varepsilon^{a b c d} \theta_{c}^{\hat{+}} v_{d}^{\hat{+}}-i \theta_{c}^{\hat{+}} \theta_{d}^{\hat{+}} \partial^{a b} \mathscr{A}^{c d} & =0, \\
\nabla^{\hat{+}} v_{d}^{\hat{+}}+4 \theta_{d}^{\hat{+}} v^{\hat{+} 2} & =0, & \nabla^{\hat{+} \hat{+}} v^{\hat{+} 2}=0 .
\end{array}
$$

These equations fully fix the $\left(\theta_{a}^{\hat{+}}, u^{\hat{ \pm} A}\right)$ dependence of the coefficients and we obtain the rather simple expression for $V^{\hat{-\mathcal{A}}}$ in the approximation considered:

$$
V^{\hat{-}} \hat{-}=i \theta_{a}^{\hat{-}} \theta_{b}^{\hat{-}} \mathscr{A}^{a b}+\frac{1}{3} \Psi^{\hat{-} 3 d} \theta_{a}^{\hat{+}}\left[\mathscr{D}^{a b}, \mathscr{D}_{d b}\right]+\frac{i}{12} \Psi^{\hat{-} 4} \theta_{a}^{\hat{+}} \theta_{b}^{\hat{+}}\left[\mathscr{D}^{a c},\left[\mathscr{D}^{b d}, \mathscr{D}_{c d}\right]\right]
$$

where $\mathscr{D}_{a b}=\partial_{a b}+\mathscr{A}_{a b}=\frac{1}{2} \varepsilon_{a b c d} \mathscr{D}^{c d}$ and

$$
\left[\mathscr{D}_{a b}, \mathscr{D}_{c d}\right]=\mathscr{D}_{a b} \mathscr{A}_{c d}-\partial_{c d} \mathscr{A}_{a b} .
$$


In what follows we will be interested in the on-shell form of the invariants. The coefficients in (7.5) are simplified on shell and can be expressed through the spinor superfield strengths $W^{ \pm a}$. We can use one of the equivalent forms of the equations of motion for $W^{ \pm a}$,

$$
D_{a}^{+} W^{-b}=-\mathscr{D}_{a}^{-} W^{+b},
$$

and represent, using the relation (3.15), the second coefficient in the expansion (7.5) as

$$
\left[\mathscr{D}^{a b}, \mathscr{D}_{d b}\right]=\frac{1}{2} D_{d}^{+} W^{-a}=-\frac{1}{2} \mathscr{D}_{d}^{-} W^{+a} .
$$

Also, after simple algebra, using the on-shell cyclic identity (3.32), we derive

$$
\left[\mathscr{D}^{[a c},\left[\mathscr{D}^{b] d}, \mathscr{D}_{c d}\right]\right]=\frac{i}{2}\left\{W^{+[a}, W^{-b]}\right\} .
$$

Now one can compute the total vector connection

$$
\mathscr{V}^{a b} \rightarrow \mathscr{A}^{a b}-\frac{i}{2} \varepsilon^{a b c d} \theta_{c}^{\hat{-}} \theta_{g}^{\hat{+}} D_{d}^{+} W^{-g}+\frac{i}{4} \varepsilon^{a b c d} \theta_{c}^{\hat{-}} \theta_{d}^{\hat{-}} \theta_{g}^{\hat{+}} \theta_{f}^{\hat{+}}\left\{W^{+g}, W^{-f}\right\},
$$

and also the rest of the harmonic projections of the covariant superfield strength

$$
\begin{aligned}
\phi^{+\hat{-}} \rightarrow & -\theta_{a}^{\hat{-}} W^{+a}-i \theta_{b}^{\hat{-}} \theta_{c}^{\hat{-}} \theta_{a}^{\hat{+}} \mathscr{D}^{b c} W^{+a}-\frac{1}{6} \Psi^{\hat{-} 3 d} \theta_{a}^{\hat{+}} \theta_{b}^{\hat{+}} D_{d}^{+}\left\{W^{-a}, W^{+b}\right\} \\
& +\frac{1}{24 \cdot 6} \Psi^{\hat{-} 4} \Psi^{\hat{+} 3 d} \varepsilon_{a b c d}\left[\left\{W^{+a}, W^{-b}\right\}, W^{+c}\right], \\
\phi^{-\hat{-}} \rightarrow & -\theta_{a}^{\hat{-}} W^{-a}-i \theta_{b}^{\hat{-}} \theta_{c}^{\hat{-}} \theta_{a}^{\hat{+}} \mathscr{D}^{b c} W^{-a}-\frac{1}{6} \Psi^{\hat{-} 3 d} \theta_{a}^{\hat{+}} \theta_{b}^{\hat{+}}\left[D_{d}^{+} W^{-a}, W^{-b}\right] \\
& +\frac{1}{24 \cdot 6} \Psi^{\hat{-4}} \Psi^{\hat{+} 3 d} \varepsilon_{a b c d}\left[\left\{W^{+a}, W^{-b}\right\}, W^{-c}\right] .
\end{aligned}
$$

As the first example of passing to $\mathscr{N}=(1,0)$ superfield notation, we rewrite the on-shell invariant (5.22). Using the representation

$$
d \hat{\zeta}_{I I}^{(-4,0)}=d \zeta^{(-4)} d \hat{u}\left(D^{\hat{-}}\right)^{4}\left(D^{\hat{+}}\right)^{4},
$$

and the relations $\left(D^{\hat{ \pm}}\right)^{4} \Psi^{\hat{+} 4}=-4$ ! (plus a total $x$-derivative), we find

$$
\begin{aligned}
S_{(10)} \rightarrow & \int d \zeta^{(-4)} \mathscr{L}_{(10)}^{+4}, \\
\mathscr{L}_{(10)}^{+4}= & \varepsilon_{a b c d} \operatorname{Tr}\left\{\left(W^{+a} W^{+b} W^{+e}+W^{+e} W^{+a} W^{+b}\right)\left[\mathscr{D}_{e}^{-} W^{+c}, W^{+d}\right]\right. \\
& \left.-2 W^{+a} W^{+b} \mathscr{D}_{g f} W^{+c} \mathscr{D}^{g f} W^{+d}\right\} .
\end{aligned}
$$

It is straightforward to check the implicit on-shell analyticity of $\mathscr{L}_{(10)}^{+4}$,

$$
D_{a}^{+} \mathscr{L}_{(10)}^{+4}=0 .
$$

This highlights the property that the $\mathbf{d}=\mathbf{1 0}$ single-trace invariant in the form (5.22) is both $\mathscr{N}=(1,0)$ and $\mathscr{N}=(0,1)$ supersymmetric only on shell. Note that the same Lagrangian, up to a total derivative, can be rewritten as the trace of two (anti)commutators

$$
\begin{aligned}
\mathscr{L}_{(10)}^{+4}= & \frac{1}{3} \varepsilon_{a b c d} \operatorname{Tr}\left(\left[D_{f}^{+} W^{-a}, W^{+b}\right]\left[\left\{W^{+c}, W^{+f}\right\}, W^{+d}\right]\right. \\
& \left.+\left\{W^{+a}, \mathscr{D}_{g f} W^{+b}\right\}\left\{W^{+c}, \mathscr{D}^{g f} W^{+d}\right\}\right),
\end{aligned}
$$

from which it follows, in particular, that this invariant vanishes in the abelian limit. 
Analogously, one can find the $\mathscr{N}=(1,0)$ superfield core of the on-shell double-trace invariant (5.24):

$$
\begin{aligned}
\mathscr{S}_{(10) I I}^{(2)} & \rightarrow \int d \zeta^{(-4)} \mathscr{L}_{(10) I I}^{+4}, \\
\mathscr{L}_{(10) I I}^{+4} & =\varepsilon_{a b c d} \operatorname{Tr}\left(W^{+a} W^{+b}\right) \operatorname{Tr}\left(\mathscr{D}_{g f} W^{+c} \mathscr{D}^{g f} W^{+d}+W^{+f}\left[D_{f}^{+} W^{-c}, W^{+d}\right]\right) \\
& \simeq-2 \varepsilon_{a b c d} \operatorname{Tr}\left(\mathscr{D}_{g f} W^{+a} W^{+b}\right) \operatorname{Tr}\left(\mathscr{D}^{g f} W^{+c} W^{+d}\right) .
\end{aligned}
$$

The on-shell analyticity of this Lagrangian can also be easily checked. In the abelian limit this expression is reduced to

$$
\sim \varepsilon_{a b c d} \partial_{g f} W^{+a} W^{+b} \partial^{g f} W^{+c} W^{+d},
$$

that is a total derivative, when taking account of the condition (3.34).

Let us proceed to $\mathbf{d}=\mathbf{1 2}$ invariants. We use $d \hat{Z}=d Z d \hat{u}\left(D^{-}\right)^{4}\left(D^{+}\right)^{4}$. For the singletrace invariants without derivatives, $S_{(12) I}^{(1)}$ and $S_{(12) I I}^{(1)}$ defined in (6.10), we find

$$
\begin{aligned}
S_{(12) I, I I}^{(1)} \rightarrow & \int d Z L_{(12) I, I I}^{(1)}, \\
L_{(12) I}^{(1)}= & -\varepsilon_{a b c d} \operatorname{Tr}\left\{\left(W^{+a} W^{+b} W^{-f} W^{-c}+W^{-a} W^{-f} W^{+b} W^{+c}\right.\right. \\
& \left.+W^{-a} W^{+b} W^{+c} W^{-f}+W^{-f} W^{+a} W^{+b} W^{-c}\right) D_{f}^{+} W^{-d} \\
& \left.+2 W^{+a} W^{+b} \mathscr{D}_{f g} W^{-c} \mathscr{D}^{f g} W^{-d}\right\} \\
= & -2 \varepsilon_{a b c d} \operatorname{Tr}\left\{\left(W^{-a} W^{+b} W^{+c} W^{-f}+W^{-f} W^{+a} W^{+b} W^{-c}\right) D_{f}^{+} W^{-d}\right. \\
& \left.+W^{+a} W^{+b} \mathscr{D}_{f g} W^{-c} \mathscr{D}^{f g} W^{-d}\right\} \\
L_{(12) I I}^{(1)}= & 2 \varepsilon_{a b c d} \operatorname{Tr}\left\{\left(W^{-a} W^{+b} W^{-f} W^{+c}+W^{+a} W^{-f} W^{+b} W^{-c}\right) D_{f}^{+} W^{-d}\right. \\
& \left.-W^{+a} \mathscr{D}_{f g} W^{-b} W^{+c} \mathscr{D}^{f g} W^{-d}\right\} .
\end{aligned}
$$

When passing to another representation of $L_{(12) I}^{(1)}$ in (7.19), we integrated by parts with respect to $D_{f}^{+}$and used the equations of motion once again.

Quite analogously, one can compute the $\mathscr{N}=(1,0)$ SYM cores of the double-trace invariants $S_{(12) I}^{(2)}$ and $S_{(12) I I}^{(2)}$. Integrating by parts and using the on-shell condition (3.33), we find

$$
\begin{aligned}
S_{(12) I, I I}^{(2)} \rightarrow & \int d Z L_{(12) I, I I}^{(2)}, \\
L_{(12) I}^{(2)}= & 4 \varepsilon_{a b c d} \operatorname{Tr}\left(W^{+a} \mathscr{D}_{g f} W^{+b}\right) \operatorname{Tr}\left(W^{-c} \mathscr{D}^{g f} W^{-d}\right), \\
L_{(12) I I}^{(2)}= & -2 \varepsilon_{a b c d}\left\{\operatorname{Tr}\left(W^{+a} \mathscr{D}_{f g} W^{-b}\right) \operatorname{Tr}\left(W^{+c} \mathscr{D}^{f g} W^{-d}\right)\right. \\
& \left.+\operatorname{Tr}\left(W^{-f} W^{+a}\right) \operatorname{Tr}\left(\left\{W^{+b}, W^{-c}\right\} D_{f}^{+} W^{-d}\right)\right\} .
\end{aligned}
$$

Recall that in the abelian limit these invariants become identical to each other up to numerical coefficients. They can be reduced to the expression

$$
S_{(12)}^{(\text {abel })}=\varepsilon_{a b c d} \int d Z \partial^{g f} W^{+a} \partial_{g f} W^{+b} W^{-c} W^{-d},
$$


which is not a total derivative even on shell, with $\partial_{g f} \partial^{g f} W^{ \pm d}=0$. Hence this sort of invariants could be relevant to the Coulomb branch of the theory, with the original gauge symmetry being broken down to some abelian subgroup (e.g., Cartan subgroup).

Before going to the invariants with the spinor and vector derivatives, it will be convenient to define the set of $\mathscr{N}=(1,0) \mathrm{SYM}$ invariants in terms of which the $\mathscr{N}=(1,0) \mathrm{SYM}$ cores of the single-trace on-shell $\mathscr{N}=(1,1)$ invariants considered here admit a succinct compact expression. Using the on-shell conditions and integrating by parts with respect to various derivatives (including the harmonic ones), it is possible to show that there exist only five independent structures of this kind. It is convenient to choose them as

$$
\begin{aligned}
& E_{1}=\varepsilon_{a b c d} \operatorname{Tr} \int d Z W^{+a} W^{+b} W^{-c} W^{-f} D_{f}^{+} W^{-d}, \\
& E_{2}=\varepsilon_{a b c d} \operatorname{Tr} \int d Z W^{-a} W^{+b} W^{+c} W^{-f} D_{f}^{+} W^{-d}, \\
& E_{3}=\varepsilon_{a b c d} \operatorname{Tr} \int d Z W^{-a} W^{-f} W^{+b} W^{+c} D_{f}^{+} W^{-d}, \\
& Y_{1}=\varepsilon_{a b c d} \operatorname{Tr} \int d Z W^{+a} W^{+b} \mathscr{D}_{f g} W^{-c} \mathscr{D}^{f g} W^{-d}, \\
& Y_{2}=\varepsilon_{a b c d} \operatorname{Tr} \int d Z W^{+a} W^{-b} \mathscr{D}_{f g} W^{-c} \mathscr{D}^{f g} W^{+d} .
\end{aligned}
$$

Using the list of integrals given in appendix, the explicit expressions for the $\mathscr{N}=(1,0)$ SYM cores of the invariants $S_{I, I I}^{(1)}$ found earlier can be expressed through the independent integrals as

$$
S_{I}^{(1)} \rightarrow-2\left(E_{2}+E_{3}+Y_{1}\right), \quad S_{I I}^{(1)} \rightarrow-2\left[4 E_{1}+3\left(E_{2}+E_{3}\right)+2\left(Y_{1}+Y_{2}\right)\right] .
$$

Note that the only combination of $Y_{1}$ and $Y_{2}$ which vanishes on shell in the abelian limit is

$$
Y_{1}+2 Y_{2}
$$

It appears just in the difference $S_{I}^{(1)}-S_{I I}^{(1)}$, which can be transformed on shell to the form $\sim \int \operatorname{Tr} d \hat{Z}\left[\phi^{+\hat{+}}, \phi^{-\hat{-}}\right]^{2}$ that vanishes in the abelian limit (the superfield densities of the structures $E_{1,2,3}$ are reduced to total $D_{a}^{+}$derivatives in this limit).

Now we can proceed to the remaining invariants $S_{(12)(3)}^{(1)}$ and $S_{(12)(2)}^{(1)}$ defined in (6.22).

It will be convenient to rewrite, using various on-shell properties of $\phi^{ \pm \hat{ \pm}}$ and integrating by parts, the Lagrangian density $L_{(12)(3)}^{(1)}$ as

$$
L_{(12)(3)}^{(1)} \simeq-\operatorname{Tr}\left(\phi^{+\hat{+}} \phi^{-\hat{-}} D^{\hat{+a}} \nabla_{a}^{+} \phi^{-\hat{-}}\right),
$$

and also to take into account that

$$
\nabla_{a}^{ \pm}=\mathscr{D}_{a}^{ \pm}+\theta_{a}^{\hat{-}}\left[\phi^{ \pm \hat{+}},\right]
$$

Rather boring (albeit straightforward) calculations yield

$$
S_{(12)(3)}^{(1)} \rightarrow 2\left[8 E_{1}+7 E_{2}+E_{3}+2\left(Y_{1}+2 Y_{2}\right)\right] .
$$


We observe that here just the combination (7.30) appears, so the $\mathscr{N}=(1,0)$ core of the 3 -superfield invariant is on-shell vanishing in the abelian limit.

One way to calculate the $\mathscr{N}=(1,0)$ limit of the Lagrangian density of the remaining invariant $S_{(12)(2)}^{(1)}$ is to use the explicit form of the vector connection (7.10). Another, simpler way is to note that this Lagrangian can be conveniently rearranged as

$$
L_{(12)(2)}^{(1)} \simeq-\frac{1}{4} \operatorname{Tr}\left(D^{\hat{+} a} D^{\hat{+} b} \phi^{+\hat{-}} \nabla_{b}^{-} \nabla_{a}^{-} \phi^{+\hat{-}}\right) .
$$

Once again, rather cumbersome time-consuming calculations finally yield the expression

$$
S_{(12)(2)}^{(1)} \rightarrow 4\left(2 E_{1}+E_{2}+E_{3}\right)+2\left(Y_{1}+2 Y_{2}\right),
$$

from which it follows, in particular, that this invariant also does not contribute to the abelian limit.

Finally, to give a feeling how these invariants look in terms of components, we will present the component form of the abelian $\mathscr{N}=(1,0)$ superfield Lagrangian (7.23), with all fields, except for the gauge field $A_{a b}(x)$, being omitted. The corresponding form of $V^{++}$ in WZ gauge and in the analytic basis is just

$$
V^{++}=i \theta^{+a} \theta^{+b} A_{a b}
$$

The relevant non-analytic harmonic connection $V^{--}$is easily restored from the abelian version of the flatness condition (3.3):

$$
V^{--}=i \theta^{-a} \theta^{-b} A_{a b}+\Psi_{d}^{-3} \theta^{+c} \mathscr{F}_{c}^{d}, \quad \mathscr{F}_{c}^{d}:=\frac{1}{6} \varepsilon^{a b f d}\left(\partial_{a b} A_{f c}-\partial_{f c} A_{a b}\right),
$$

whence

$$
W^{+a}=-\frac{1}{6} \varepsilon^{a b c d} D_{b}^{+} D_{c}^{+} D_{d}^{+} V^{--}=-\theta^{+b} \mathscr{F}_{b}^{a} .
$$

That part of $W^{-a}=D^{--} W^{+a}$ which contributes to the Berezin integral in (7.23) can also be easily found

$$
W^{-a} \rightarrow-i \theta^{-b} \theta^{-c} \theta^{+d} \partial_{b c} \mathscr{F}_{d}^{a} .
$$

Then it is straightforward to perform the Berezin integration and to find

$$
S_{(12)}^{(\text {abel })}=-2 \varepsilon^{a b c d} \varepsilon_{e f g h} \int d^{6} x\left(\partial^{l t} \mathscr{F}_{a}^{e} \partial_{l t} \mathscr{F}_{b}^{f}\right)\left(\partial^{m n} \mathscr{F}_{c}^{g} \partial_{m n} \mathscr{F}_{d}^{h}\right) .
$$

The same expression in the vector notation looks rather bulky. Passing to the vector notation according to eqs. (2.6), (2.7), we obtain ${ }^{9}$

$$
\mathscr{F}_{b}^{a}=-\frac{1}{12}\left(\sigma^{M N}\right)_{b}^{a} \mathscr{F}_{M N}, \quad \mathscr{F}_{M N}=\partial_{M} A_{N}-\partial_{N} A_{M}=\frac{3}{2} \mathscr{F}_{b}^{a}\left(\sigma_{M N}\right)_{a}^{b},
$$

and rewrite, up to a numerical coefficient, the action (7.39) with the help of relations (2.5) as

$$
\begin{aligned}
S_{(12)}^{(\text {abel })} \sim & \int d^{6} x\left[\left(\partial \mathscr{F}^{M N} \cdot \partial \mathscr{F}_{M N}\right)^{2}+2\left(\partial \mathscr{F}^{M N} \cdot \partial \mathscr{F}^{S T}\right)\left(\partial \mathscr{F}_{M N} \cdot \partial \mathscr{F}_{S T}\right)\right. \\
& \left.-4\left(\partial \mathscr{F}^{M N} \cdot \partial \mathscr{F}^{S T}\right)\left(\partial \mathscr{F}_{M S} \cdot \partial \mathscr{F}_{N T}\right)-8\left(\partial \mathscr{F}_{N}^{M} \cdot \partial \mathscr{F}_{M T}\right)\left(\partial \mathscr{F}^{S N} \cdot \partial \mathscr{F}_{S}^{T}\right)\right],
\end{aligned}
$$

where $\partial \mathscr{F}^{M N} \cdot \partial \mathscr{F}_{M N}:=\partial^{L} \mathscr{F}^{M N} \partial_{L} \mathscr{F}_{M N}$, etc.

\footnotetext{
${ }^{9}$ The gauge potentials $A_{M}$ are imaginary with our conventions.
} 
It is interesting that there exist no $\mathbf{d}=\mathbf{1 2}$ invariants which could yield, in the abelian gauge theory limit, the expression like $\sim F^{6}$, as distinct from the $\mathbf{d}=\mathbf{8}$ invariants which contain $F^{4}$ term in such a limit [26]. It is likely that the abelian $F^{6}$ terms do not admit even $\mathscr{N}=(1,0)$ completion.

\section{Summary and outlook}

In this paper we continued the applications of the on-shell $6 D, \mathscr{N}=(1,1)$ HSS for constructing higher-derivative invariants of the maximally supersymmetric $6 D$ SYM theory. We have explicitly constructed new, dimension $\mathbf{d}=\mathbf{1 2}$, single-trace invariants (6.22) and the double-trace invariants (6.23), studied their $\mathscr{N}=(1,0) \mathrm{SYM}$ cores and the component gauge-field structure of their abelian limit. All these invariants are given by integrals over the total bi-harmonic $6 D$ superspace and we did not find any "no-go"-type conjectures which would forbid the appearance of the four-loop logarithmic divergences of such a form within the off-shell $6 D, \mathscr{N}=(1,0)$ HSS supergraph calculations in the quantum $\mathscr{N}=(1,1)$ SYM theory. The leading in momenta contributions should come from (6.23) and the invariants in the first line of (6.22), while the rest of the single-trace invariants, including additional derivatives, should be responsible for some sub-leading terms (divergent and/or finite) in the relevant order of the total effective action.

It would be interesting to look for the invariants of the dimension $\mathbf{d}=\mathbf{1 4}$ and $\mathbf{d}=\mathbf{1 6}$ corresponding to the five- and six-loop contributions. The leading terms should be a direct generalization of those in (6.22) and (6.23). E.g., one of the possible candidates for such invariants of the dimension $\mathbf{d}=\mathbf{1 4}$ reads

$$
\sim \operatorname{Tr} \int d \hat{Z}\left(\phi^{+\hat{+}}\right)^{3}\left(\phi^{-\hat{-}}\right)^{3}
$$

and it seems that finding the full set of independent on-shell invariants of this sort could be a comparatively easy straightforward task. On the other hand, according to the arguments of ref. [28], already at the dimension $\mathbf{d}=\mathbf{1 6}$ there should be taken into account deformations of the conventional mass shell associated with the superfield equations of motion (3.29) and (3.28). So the formalism of the on-shell $6 D, \mathscr{N}=(1,1)$ HSS should undergo a proper modification. It would be extremely interesting to explicitly learn how this would happen, how the initial constraints of $6 D, \mathscr{N}=(1,1)$ SYM theory are to be corrected and which geometric principle could stand behind such a modification.

\section{Acknowledgments}

E.I. thanks G. Bossard, I. Buchbinder and A. Smilga for illuminating discussions of various issues related to the subject of this paper. The work of E.I. was partly supported by Russian Scientific Foundation, project No. 21-12-00129.

\section{A Some integrals}

Here we express, through the basic structures defined in (7.24)-(7.26), various $\mathscr{N}=(1,0)$ integrals encountered when calculating the $\mathscr{N}=(1,0)$ SYM limit of the $\mathscr{N}=(1,1)$ 
invariants in section 7 . While transforming the relevant integrands, we integrate by parts and use the on-shell conditions $D_{a}^{+} W^{+d}=\mathscr{D}_{a}^{-} W^{-d}=0, D_{a}^{+} W^{-d}=-\mathscr{D}_{a}^{-} W^{+d}, D_{a}^{+} W^{-a}=$ $0, \nabla^{ \pm \pm} W^{ \pm a}=0, \nabla^{ \pm \pm} W^{\mp a}=W^{ \pm a}$. In this way we derive the following list of the on-shell $\mathscr{N}=(1,0)$ SYM integrals

$$
\begin{aligned}
& \varepsilon_{a b c d} \operatorname{Tr} \int d Z W^{+a} W^{-f} W^{+b} W^{-c} D_{f}^{+} W^{-d}=-\left(E_{1}+E_{2}+E_{3}\right), \\
& \varepsilon_{a b c d} \operatorname{Tr} \int d Z W^{+a} W^{-f} W^{-b} W^{+c} D_{f}^{+} W^{-d}=E_{1}+E_{2}, \\
& \varepsilon_{a b c d} \operatorname{Tr} \int d Z W^{+a} W^{-b} W^{+c} W^{-f} D_{f}^{+} W^{-d}=-\left(E_{1}+E_{2}\right), \\
& \varepsilon_{a b c d} \operatorname{Tr} \int d Z W^{-a} W^{+b} W^{-f} W^{+c} D_{f}^{+} W^{-d}=-\left(E_{1}+E_{2}+E_{3}\right), \\
& \varepsilon_{a b c d} \operatorname{Tr} \int d Z W^{-f} W^{+a} W^{+b} W^{-c} D_{f}^{+} W^{-d}=E_{3}, \\
& \varepsilon_{a b c d} \operatorname{Tr} \int d Z W^{-f} W^{-a} W^{+b} W^{+c} D_{f}^{+} W^{-d}=E_{1} .
\end{aligned}
$$

Open Access. This article is distributed under the terms of the Creative Commons Attribution License (CC-BY 4.0), which permits any use, distribution and reproduction in any medium, provided the original author(s) and source are credited.

\section{References}

[1] A.A. Tseytlin, On nonAbelian generalization of Born-Infeld action in string theory, Nucl. Phys. B 501 (1997) 41 [hep-th/9701125] [INSPIRE].

[2] C. Cheung and D. O'Connell, Amplitudes and Spinor-Helicity in Six Dimensions, JHEP 07 (2009) 075 [arXiv: 0902.0981] [INSPIRE].

[3] J. Plefka, T. Schuster and V. Verschinin, From Six to Four and More: Massless and Massive Maximal Super Yang-Mills Amplitudes in $6 d$ and $4 d$ and their Hidden Symmetries, JHEP 01 (2015) 098 [arXiv: 1405.7248] [INSPIRE].

[4] T. Dennen, Y.-t. Huang and W. Siegel, Supertwistor space for $6 D$ maximal super Yang-Mills, JHEP 04 (2010) 127 [arXiv:0910.2688] [InSPIRE].

[5] Y.-t. Huang and A.E. Lipstein, Amplitudes of $3 D$ and $6 D$ Maximal Superconformal Theories in Supertwistor Space, JHEP 10 (2010) 007 [arXiv:1004.4735] [INSPIRE].

[6] Z. Bern, J.J. Carrasco, T. Dennen, Y.-t. Huang and H. Ita, Generalized Unitarity and Six-Dimensional Helicity, Phys. Rev. D 83 (2011) 085022 [arXiv:1010.0494] [INSPIRE].

[7] Z. Bern, J.J.M. Carrasco and H. Johansson, New Relations for Gauge-Theory Amplitudes, Phys. Rev. D 78 (2008) 085011 [arXiv:0805.3993] [InSPIRE].

[8] Z. Bern, L.J. Dixon and V.A. Smirnov, Iteration of planar amplitudes in maximally supersymmetric Yang-Mills theory at three loops and beyond, Phys. Rev. D 72 (2005) 085001 [hep-th/0505205] [INSPIRE].

[9] Z. Bern, J.J.M. Carrasco, L.J. Dixon, H. Johansson and R. Roiban, The Complete Four-Loop Four-Point Amplitude in $N=4$ Super-Yang-Mills Theory, Phys. Rev. D 82 (2010) 125040 [arXiv: 1008.3327] [INSPIRE]. 
[10] Z. Bern, J.J.M. Carrasco, L.J. Dixon, H. Johansson and R. Roiban, Simplifying Multiloop Integrands and Ultraviolet Divergences of Gauge Theory and Gravity Amplitudes, Phys. Rev. D 85 (2012) 105014 [arXiv:1201.5366] [INSPIRE].

[11] N. Berkovits, M.B. Green, J.G. Russo and P. Vanhove, Non-renormalization conditions for four-gluon scattering in supersymmetric string and field theory, JHEP 11 (2009) 063 [arXiv:0908.1923] [INSPIRE].

[12] J. Bjornsson and M.B. Green, 5 loops in 24/5 dimensions, JHEP 08 (2010) 132 [arXiv: 1004.2692] [INSPIRE].

[13] J. Bjornsson, Multi-loop amplitudes in maximally supersymmetric pure spinor field theory, JHEP 01 (2011) 002 [arXiv: 1009.5906] [INSPIRE].

[14] L.V. Bork, D.I. Kazakov, M.V. Kompaniets, D.M. Tolkachev and D.E. Vlasenko, Divergences in maximal supersymmetric Yang-Mills theories in diverse dimensions, JHEP 11 (2015) 059 [arXiv: 1508.05570] [INSPIRE].

[15] G. Bossard, P.S. Howe and K.S. Stelle, The Ultra-violet question in maximally supersymmetric field theories, Gen. Rel. Grav. 41 (2009) 919 [arXiv:0901.4661] [INSPIRE].

[16] G. Bossard, P.S. Howe and K.S. Stelle, A Note on the UV behaviour of maximally supersymmetric Yang-Mills theories, Phys. Lett. B 682 (2009) 137 [arXiv:0908.3883] [INSPIRE].

[17] P.S. Howe, G. Sierra and P.K. Townsend, Supersymmetry in Six-Dimensions, Nucl. Phys. B 221 (1983) 331 [INSPIRE].

[18] J. Koller, A six-dimensional superspace approach to extended superfields, Nucl. Phys. B 222 (1983) 319 [INSPIRE].

[19] A. Galperin, E. Ivanov, S. Kalitsyn, V. Ogievetsky and E. Sokatchev, Unconstrained $N=2$ Matter, Yang-Mills and Supergravity Theories in Harmonic Superspace, Class. Quant. Grav. 1 (1984) 469 [Erratum ibid. 2 (1985) 127] [INSPIRE].

[20] A.S. Galperin, E.A. Ivanov, V.I. Ogievetsky and E.S. Sokatchev, Harmonic superspace, in Cambridge Monographs on Mathematical Physics, Cambridge University Press, Cambridge U.K. (2007).

[21] P.S. Howe, K.S. Stelle and P.C. West, $N=1 d=6$ harmonic superspace, Class. Quant. Grav. 2 (1985) 815 [INSPIRE].

[22] B.M. Zupnik, Six-dimensional Supergauge Theories in the Harmonic Superspace, Sov. J. Nucl. Phys. 44 (1986) 512 [Yad. Fiz. 44 (1986) 794] [INSPIRE].

[23] P.S. Howe and K.S. Stelle, Ultraviolet Divergences in Higher Dimensional Supersymmetric Yang-Mills Theories, Phys. Lett. B 137 (1984) 175 [InSPIRE].

[24] P.S. Howe and K.S. Stelle, Supersymmetry counterterms revisited, Phys. Lett. B 554 (2003) 190 [hep-th/0211279] [INSPIRE].

[25] G. Bossard, P.S. Howe, U. Lindström, K.S. Stelle and L. Wulff, Integral invariants in maximally supersymmetric Yang-Mills theories, JHEP 05 (2011) 021 [arXiv:1012.3142] [INSPIRE].

[26] G. Bossard, E. Ivanov and A. Smilga, Ultraviolet behavior of 6D supersymmetric Yang-Mills theories and harmonic superspace, JHEP 12 (2015) 085 [arXiv: 1509.08027] [INSPIRE]. 
[27] I.L. Buchbinder, E.A. Ivanov and V.A. Ivanovskiy, New bi-harmonic superspace formulation of $4 D, \mathscr{N}=4 S Y M$ theory, JHEP 04 (2021) 010 [arXiv:2012.09669] [INSPIRE].

[28] A. Smilga, Ultraviolet divergences in non-renormalizable supersymmetric theories, Phys. Part. Nucl. Lett. 14 (2017) 245 [arXiv:1603.06811] [INSPIRE].

[29] I.L. Buchbinder, E.A. Ivanov, B.S. Merzlikin and K.V. Stepanyantz, One-loop divergences in the $6 D, \mathscr{N}=(1,0)$ Abelian gauge theory, Phys. Lett. B 763 (2016) 375 [arXiv:1609.00975] [INSPIRE].

[30] I.L. Buchbinder, E.A. Ivanov, B.S. Merzlikin and K.V. Stepanyantz, One-loop divergences in $6 D, \mathscr{N}=(1,0) S Y M$ theory, JHEP 01 (2017) 128 [arXiv:1612.03190] [INSPIRE].

[31] I.L. Buchbinder, E.A. Ivanov, B.S. Merzlikin and K.V. Stepanyantz, Supergraph analysis of the one-loop divergences in $6 D, \mathscr{N}=(1,0)$ and $\mathscr{N}=(1,1)$ gauge theories, Nucl. Phys. $B$ 921 (2017) 127 [arXiv: 1704.02530] [INSPIRE].

[32] E.A. Ivanov, A.V. Smilga and B.M. Zupnik, Renormalizable supersymmetric gauge theory in six dimensions, Nucl. Phys. B 726 (2005) 131 [hep-th/0505082] [INSPIRE].

[33] I.L. Buchbinder and N.G. Pletnev, Construction of $6 D$ supersymmetric field models in $N=(1,0)$ harmonic superspace, Nucl. Phys. B 892 (2015) 21 [arXiv:1411.1848] [INSPIRE].

[34] S.M. Kuzenko, J. Novak and I.B. Samsonov, The anomalous current multiplet in $6 D$ minimal supersymmetry, JHEP 02 (2016) 132 [arXiv:1511.06582] [INSPIRE].

[35] S.M. Kuzenko, J. Novak and I.B. Samsonov, Chiral anomalies in six dimensions from harmonic superspace, JHEP 11 (2017) 145 [arXiv:1708.08238] [INSPIRE].

[36] I.L. Buchbinder, E.A. Ivanov, B.S. Merzlikin and K.V. Stepanyantz, On the two-loop divergences of the 2-point hypermultiplet supergraphs for $6 D, \mathscr{N}=(1,1) S Y M$ theory, Phys. Lett. B 778 (2018) 252 [arXiv:1711.11514] [INSPIRE].

[37] I.L. Buchbinder, E.A. Ivanov, B.S. Merzlikin and K.V. Stepanyantz, On the two-loop divergences in $6 D, \mathscr{N}=(1,1)$ SYM theory, Phys. Lett. B 820 (2021) 136516 [arXiv: 2104.14284] [INSPIRE].

[38] A.A. Tseytlin and K. Zarembo, Magnetic interactions of D-branes and Wess-Zumino terms in superYang-Mills effective actions, Phys. Lett. B 474 (2000) 95 [hep-th/9911246] [INSPIRE].

[39] K.A. Intriligator, Anomaly matching and a Hopf-Wess-Zumino term in $6 d, N=(2,0)$ field theories, Nucl. Phys. B 581 (2000) 257 [hep-th/0001205] [INSPIRE].

[40] P.C. Argyres, A.M. Awad, G.A. Braun and F.P. Esposito, Higher derivative terms in $N=2$ supersymmetric effective actions, JHEP 07 (2003) 060 [hep-th/0306118] [INSPIRE]. 